\title{
Random Variate Generation for Exponential and Gamma Tilted Stable Distributions
}

\author{
Yan $\mathrm{Qu}^{*}$ \\ Angelos Dassios ${ }^{\dagger}$ \\ University of Warwick \\ London School of Economics \\ Hongbiao Zhao \\ Shanghai University of Finance and Economics
}

3rd February 2021

\begin{abstract}
We develop a new efficient simulation scheme for sampling two families of tilted stable distributions: exponential tilted stable (ETS) and gamma tilted stable (GTS) distributions. Our scheme is based on two-dimensional single rejection (SR). For the ETS family, its complexity is uniformly bounded over all ranges of parameters. This new algorithm outperforms all existing schemes. In particular, it is more efficient than the well-known double rejection (DR) scheme (Devroye, 2009), which is the only algorithm with uniformly bounded complexity that we can find in the current literature. Beside the ETS family, our scheme is also flexible to be further extended for generating the GTS family, which cannot easily be done by extending the DR scheme. Our algorithms are straightforward to implement, and numerical experiments and tests are conducted to demonstrate the accuracy and efficiency.
\end{abstract}

Keywords: Exponentially tilted stable distribution; Gamma tilted stable distribution; Exact Simulation Algorithms; Monte Carlo simulation; Random variate generation; Two-dimensional single rejection; Tempered stable distribution; Lévy process

\section{Introduction}

The family of positive stable distributions, which was introduced by Lévy (1925), is an important mathematical tool for capturing heavy tails of observations from reality, such as financial time

\footnotetext{
${ }^{*}$ Corresponding author, Department of Statistics, University of Warwick, Coventry CV4 7AL, United Kingdom. Email: y.qu3@1se.ac.uk

'Department of Statistics, London School of Economics, London WC2A 2AE, United Kingdom. Email: a.dassios@1se.ac.uk

${ }^{\ddagger}$ School of Statistics and Management, Shanghai University of Finance and Economics, No. 777 Guoding Road, Shanghai 200433, China. Email: h.zhao1@1se.ac.uk
} 
series of price returns. A series of influential work by Mandelbrot (1961, 1963a,b) had demonstrated its importance for potential applications in finance and economics. However, there is one crucial problem, as later pointed by many scholars, is its infinite moments, which would be especially problematic for pricing assets such as options. In order to deal with this issue, the tail of a positive stable distribution should be tilted (or tempered); see discussions in Carr and Wu (2003) and $\mathrm{Wu}$ (2006). A very popular version of the tilted stable distribution is the so-called exponentially tilted stable (ETS) distribution, which was initially proposed by Tweedie (1984) and Hougaard (1986). It plays a key role in mathematical statistics, as a model for randomness used by Bayesians, and in economic models (Devroye, 2009). Furthermore, the family of ETS distributions has become a fundamental component to be used to construct many useful stochastic processes, which have numerous applications in finance and many other fields. For example, ETS-driven non-Gaussian Ornstein-Uhlenbeck processes are used for modelling stochastic volatilities of asset prices and contagion risk processes, see Barndorff-Nielsen and Shephard (2002, 2003); Andrieu et al. (2010); Todorov (2015); Qu et al. (2021, 2019). More recently, ETS-driven Lévy subordinators have been adopted for modelling the stochastic-time clocks in a series of time-changed models proposed by Li and Linetsky $(2013,2014,2015)$ and Mendoza-Arriaga and Linetsky $(2014,2016)$. Besides, ETS distributions as key members of infinitely divisible distributions, are closely connected with characteristic kernels, which play an import role in machine learning applications, see Nishiyama and Fukumizu (2016).

The simulation design for sampling ETS distributions without bias has been recently brought to the attention in the literature. The most widely used and trivial algorithm probably is the simple stable rejection (SSR) scheme, which is developed by a simple combination of the well known Zolotarev's integral representation (Zolotarev, 1966) and an acceptance-rejection (A/R) scheme; see Brix (1999). Hofert (2011a) suggested a fast rejection (FR) algorithm to enhance the SSR scheme. However, the complexities ${ }^{1}$ of SSR and FR are unbounded, which obviously limit their applicability as they would become extremely inefficient for some parameter choices. To overcome this problem, Devroye (2009) developed a novel scheme based on double rejection (DR) such that the complexity is uniformly bounded. Alternatively, in this paper, we design a new scheme for ETS distributions based on two-dimensional single rejection $(\mathrm{SR})^{2}$. The complexity of our SR scheme is also uniformly bounded, and remarkably, it outperforms the DR scheme for all ranges of parameters. More precisely, the complexity of our SR scheme is roughly bounded by 4.2154 over all

\footnotetext{
${ }^{1}$ The complexity of an algorithm is the expected number of iterations before halting, see Law (2015, Ch.8). In particular for the acceptance-rejection (A/R) methodology, its complexity is exactly the associated A/R constant.

${ }^{2}$ This idea originates from the approach of distributional decomposition and transformation adopted by Dassios et al. (2018) where they tailored efficient simulation algorithms for some special ETS classes, see also Dassios et al. (2020) for this approach.
} 
parameters, which is smaller than the one for the DR scheme. Furthermore, we can easily extend our scheme for sampling gamma tilted stable (GTS) distributions, which cannot easily be done by extending DR scheme since the R distribution suggested in Devroye (2009) has been replaced by other distribution for GTS. The GTS distribution was first introduced by Barndorff-Nielsen and Shephard (2001) for modelling stochastic volatility of financial time series. The first simulation algorithm was just developed recently by Favaro et al. (2015), which is based on the decomposition for the GTS. Since our algorithm for the GTS does not depend on the ETS simulation scheme, it is much easier to set up and implement than the one in Favaro et al. (2015).

The paper is structured as follows: In Section 2, we provide preliminaries for the positive stable distribution, exponential tilted stable distribution, introduce the general two-dimensional SR framework, and develop several simulation schemes for sampling ETS distributions. In Section 3 , we analyse the performances of several proposed algorithms regarding to different choices of tilting and stability parameters, then, by optimally combining these schemes, we propose a super efficient uniformly bounded scheme to sample ETS variables over the whole range of stability and tilting parameters. In Section 4, we extend the simulation idea from ETS distributions to GTS distributions. In Section 5, extensive numerical experiments for our algorithms as well as the associated comparisons with other schemes have been carried out and reported in detail. Section 6 draws a brief conclusion for this paper.

\section{Preliminaries}

\subsection{Exponential Tilted Stable Distributions}

A positive stable random variable $S_{\alpha}$ with the stability index $\alpha \in(0,1)$ has the Laplace transform

$$
\mathbb{E}\left[e^{-v S_{\alpha}}\right]=e^{-v^{\alpha}}, \quad v \in \mathbb{R}^{+} .
$$

The density function of $S_{\alpha}$ has the well-known Zolotarev's integral representation (Zolotarev, 1986),

$$
f_{\alpha}(s)=\frac{1}{\pi} \int_{0}^{\pi} \frac{\alpha}{1-\alpha} B(u)^{\frac{1}{1-\alpha}} s^{-\frac{1}{1-\alpha}} e^{-B(u)^{\frac{1}{1-\alpha}} s^{-\frac{\alpha}{1-\alpha}}} \mathrm{d} u, \quad s \in \mathbb{R}^{+},
$$

where $B(u)$ is defined as

$$
B(u):=\frac{\sin ^{\alpha}(\alpha u) \sin ^{1-\alpha}((1-\alpha) u)}{\sin u}, \quad u \in[0, \pi] .
$$


The associated ETS random variable $S_{\alpha, \lambda}$ is defined through the exponentially tilting distribution of $S_{\alpha}$ with tilting parameter $\lambda \in \mathbb{R}^{+}$. The Laplace transform of $S_{\alpha, \lambda}$ therefore is

$$
\mathbb{E}\left[e^{-v S_{\alpha, \lambda}}\right]=e^{\lambda^{\alpha}-(\lambda+v)^{\alpha}},
$$

and the density function of $S_{\alpha, \lambda}$ is given by

$$
f_{\alpha, \lambda}(s)=e^{\lambda^{\alpha}-\lambda s} f_{\alpha}(s)=\int_{0}^{\pi} f(s, u) \mathrm{d} u,
$$

where $f(s, u)$ is the bivariate density function of $(S, U)$ in $(s, u)$ on $[0, \infty) \times[0, \pi]$, i.e.,

$$
f(s, u)=\frac{\alpha e^{\lambda^{\alpha}}}{(1-\alpha) \pi} B(u)^{\frac{1}{1-\alpha}} s^{-\frac{1}{1-\alpha}} \exp \left(-B(u)^{\frac{1}{1-\alpha}} s^{-\frac{\alpha}{1-\alpha}}-\lambda s\right) .
$$

This $S_{\alpha, \lambda}$ can not be easily simulated directly due to the Zolotarev's integral representation (2.2). However, we can use our two-dimensional A/R scheme to sample $(S, U)$ and return $S$ to sample $S_{\alpha, \lambda}$ instead.

Remark 2.1. Other papers in the literature may use an alternative parameterisation for the exponential tilted stable distribution with Laplace transform

$$
\mathbb{E}\left[e^{-v S_{\alpha, \lambda, \theta}}\right]=e^{\theta\left[\lambda^{\alpha}-(\lambda+v)^{\alpha}\right]},
$$

where $\theta \in \mathbb{R}^{+}$is a new parameter. Without loss of generality, we set $\theta=1$ in this paper, since

$$
S_{\alpha, \lambda, \theta} \stackrel{\mathcal{D}}{=} \theta^{\frac{1}{\alpha}} S_{\alpha, \lambda \theta^{\frac{1}{\alpha}}}
$$

see Devroye (2009, p.12).

\subsection{Two-Dimensional Single Rejection Scheme}

Several competing algorithms for simulating exponentially tilted stable distributions have been proposed in the literature, i.e. simple stable rejection (SSR) scheme (Brix, 1999), fast rejection (FR) scheme (Hofert, 2011a), and double rejection (DR) scheme (Devroye, 2009). These algorithms are unbiased and can produce very accurate samples. However, each of them has its own advantages and limitations. For the SSR scheme, since the expected complexity is exponentially increasing, the algorithm has a very poor acceptance rate for a large value of tilting parameter $\lambda$. For the FR scheme, it works well for a small value of $\alpha$, but its complexity is of order $\mathcal{O}\left(\lambda^{\alpha}\right)$ which is clearly 
unbounded. For the DR scheme, although the complexity is uniformly bounded, the upper bound is still large. In particular, when $\alpha$ is close to 0 , the simulation becomes much less efficient. Comparing with the SSR scheme and the FR scheme, the DR scheme is more difficult for a practitioner to implement as the procedure is rather complicated. Hence, it is of great interest to develop a simpler and more efficient algorithm with lower uniformly bounded complexity for all $\alpha \in(0,1)$ and $\lambda \in \mathbb{R}^{+}$, and this is the aim of our paper.

Given the density function of $S_{\alpha, \lambda}$ in (2.4) with the joint density function $f(s, u)$ of a bivariate variable $(S, U)$ in (2.5), we can use the two-dimensional A/R scheme to sample $(S, U)$ by choosing an appropriate bivariate envelope $\left(S^{\prime}, U^{\prime}\right)$ with density $g(s, u)$. Therefore, we can use the following general simulation framework, Algorithm 2.1, to sample the associated marginal variate $S$.

Algorithm 2.1. We have the following two-Dimensional single rejection framework

1. set $C=\sup _{s, u}\{f(s, u) / g(s, u)\}$

2. repeat \{

3. $\quad$ sample $(S, U)$ with density $g(s, u), V \sim \mathcal{U}(0,1)$

4. if $\left(V \leq \frac{f(S, U)}{C g(S, U)}\right)$ break

5. $\}$

6. return $S$

The expected complexity, which stands for the expected number of iterations before halting, of this two-dimensional single rejection (SR) scheme is the corresponding acceptance-rejection (A/R) constant $C$ in Algorithm 2.1. Hence, if we can find an appropriate bivariate envelope with a lower and uniformly bounded $C$, then this method is more suitable than the double rejection (DR) method used by Devroye (2009), as only one rejection procedure is involved within the entire simulation instead of two.

\section{Simulation Scheme for Exponential Tilted Stable Distribution}

Based on the two-dimensional SR framework in Algorithm 2.1, we design an efficient simulation algorithm to sample the exponential tilted stable distributions with uniformly bounded complexity. First of all, let us define $\operatorname{Erf}(x):=\frac{2}{\sqrt{\pi}} \int_{0}^{x} e^{-t^{2}} \mathrm{~d} t$ as the error function, $\Gamma(x):=\int_{0}^{\infty} t^{x-1} e^{-t} \mathrm{~d} t$ as the 
gamma function, and denote $\mathcal{N}\left(\mu, \sigma^{2}, \mathrm{lb}=0, \mathrm{ub}=\pi\right)^{3}$ as the truncated normal distribution with mean $\mu \in \mathbb{R}$ and variance $\sigma^{2} \in \mathbb{R}^{+}$within the domain $[0, \pi]$. The details of the new simulation scheme for ETS distributions is provided in Algorithm 3.1 below.

Algorithm 3.1. The two-Dimensional single rejection algorithm for $S_{\alpha, \lambda}$ is provided as follows,

1. set $R=\operatorname{Erf}\left(\sqrt{\alpha(1-\alpha) \lambda^{\alpha} \pi^{2} / 2}\right), C_{1}=\frac{\Gamma\left(\alpha \lambda^{\alpha}\right) e^{\alpha \lambda^{\alpha}-1}}{\left(\alpha \lambda^{\alpha}\right)^{\lambda^{\alpha}}}\left(\frac{\alpha}{1-\alpha}+\alpha \lambda^{\alpha}\right)^{\lambda^{\alpha}(1-\alpha)+1}, C_{2}=$ $\frac{\Gamma\left((1-\alpha) \lambda^{\alpha}+1\right) e^{(1-\alpha) \lambda^{\alpha}}}{\left((1-a) \lambda^{\alpha}\right)^{(1-\alpha) \lambda^{\alpha}}}, C_{3}=\frac{\Gamma\left(\alpha \lambda^{\alpha}+1\right) e^{\alpha \lambda^{\alpha}-1}\left(\alpha \lambda^{\alpha}\right)^{-\alpha \lambda^{\alpha}}}{\sqrt{2 \pi \alpha(1-\alpha) \lambda^{\alpha}}\left(1+1 /\left[(1-\alpha) \lambda^{\alpha}\right]\right)^{-1-(1-\alpha) \lambda^{\alpha}}}, C_{4}=\frac{\Gamma\left((1-\alpha) \lambda^{\alpha}+1\right) e^{(1-\alpha) \lambda^{\alpha}}}{\sqrt{2 \pi \alpha(1-\alpha) \lambda^{\alpha}}\left((1-\alpha) \lambda^{\alpha}\right)^{(1-\alpha) \lambda^{\alpha}}}$

2. if $\left(C_{1}=\min \left\{C_{1}, C_{2}, C_{3}, C_{4}\right\}\right)$ \{

\section{3. repeat \{}

4. $\quad$ sample $U \sim \mathcal{U}[0, \pi], X \sim \Gamma\left(\alpha \lambda^{\alpha}, 1\right), V \sim \mathcal{U}[0,1]$; set $S=X / \lambda$

5. $\quad$ if $\left(V \leq \frac{\alpha e^{\lambda^{\alpha}} \Gamma\left(\alpha \lambda^{\alpha}\right)}{1-\alpha} B(U)^{\frac{1}{1-\alpha}} \lambda^{\frac{\alpha}{1-\alpha}} X^{-\frac{\alpha}{1-\alpha}-\alpha \lambda^{\alpha}} e^{-B(U)^{\frac{1}{1-\alpha}} \lambda^{\frac{\alpha}{1-\alpha}} X^{-\frac{\alpha}{1-\alpha}}} / C_{1}\right)$ break

6. $\}$

7. $\}$

8. if $\left(C_{2}=\min \left\{C_{1}, C_{2}, C_{3}, C_{4}\right\}\right)$ f

9. repeat \{

10. $\quad$ sample $U \sim \mathcal{U}[0, \pi], Z \sim \Gamma\left((1-\alpha) \lambda^{\alpha}+1,1\right), V \sim \mathcal{U}[0,1]$; set $S=B(U)^{\frac{1}{\alpha}} Z^{-\frac{1-\alpha}{\alpha}}$

11. if $\left(V \leq e^{\lambda^{\alpha}} \Gamma\left((1-\alpha) \lambda^{\alpha}+1\right) Z^{-(1-\alpha) \lambda^{\alpha}} e^{-\lambda B(U)^{\frac{1}{\alpha}} Z^{-\frac{1-\alpha}{\alpha}}} / C_{2}\right)$ break

12. 1

13. $\}$

14. if $\left(C_{3}=\min \left\{C_{1}, C_{2}, C_{3}, C_{4}\right\}\right)\{$

15. $\operatorname{repeat}_{\{}$

16. sample $U \sim \mathcal{N}\left(\mu=0, \sigma^{2}=\left[\alpha(1-\alpha) \lambda^{\alpha}\right]^{-1}, l b=0, u b=\pi\right)$

17. $\quad$ sample $X \sim \Gamma\left(\alpha \lambda^{\alpha}, 1\right), V \sim \mathcal{U}[0,1]$; set $S=X / \lambda$

18. $\quad$ if $\left(V \leq \frac{R \alpha e^{\lambda^{\alpha}} \Gamma\left(\alpha \lambda^{\alpha}\right) \lambda^{\frac{\alpha}{1-\alpha}} B(U)^{\frac{1}{1-\alpha}}}{C_{3}(1-\alpha) \sqrt{2 \pi \alpha(1-\alpha) \lambda^{\alpha}} X^{\frac{\alpha}{1-\alpha}+\alpha \lambda^{\alpha}}} e^{-\left(\lambda B(u)^{\frac{1}{\alpha}} X^{-1}\right)^{\frac{\alpha}{1-\alpha}}+\frac{\alpha(1-\alpha) \lambda^{\alpha} U^{2}}{2}}\right)$ break

19. $\}$

20. $\}$

3 "lb" stands for the lower bound and "ub" stands for the upper bound 
21. if $\left(C_{4}=\min \left\{C_{1}, C_{2}, C_{3}, C_{4}\right\}\right)\{$

\section{2. repeat \{}

23. $\quad$ sample $U \sim \mathcal{N}\left(\mu=0, \sigma^{2}=\left[\alpha(1-\alpha) \lambda^{\alpha}\right]^{-1}, l b=0, u b=\pi\right)$

24. $\quad$ sample $Z \sim \Gamma\left((1-\alpha) \lambda^{\alpha}+1,1\right), V \sim \mathcal{U}[0,1]$; set $S=B(U)^{\frac{1}{\alpha}} Z^{-\frac{1-\alpha}{\alpha}}$

25. if $\left(V \leq \frac{R e^{\lambda^{\alpha}} \Gamma\left((1-\alpha) \lambda^{\alpha}+1\right)}{C_{4} \sqrt{2 \pi \alpha(1-\alpha) \lambda^{\alpha}} Z^{(1-\alpha) \lambda^{\alpha}}} e^{-\lambda B(U)^{\frac{1}{\alpha}} Z^{-\frac{1-\alpha}{\alpha}}+\frac{\alpha(1-\alpha) \lambda^{\alpha} U^{2}}{2}}\right)$ break

26. $\}$

27. $\}$

\section{8. return $S$}

Proof. According to (2.2) and (2.4), for

$$
X=\lambda S_{\alpha, \lambda}
$$

the density of the random variable $X$ is specified by

$$
f_{X}(x)=\frac{1}{\pi} \int_{0}^{\pi} \frac{\alpha e^{\lambda^{\alpha}}}{1-\alpha} B(u)^{\frac{1}{1-\alpha}} \lambda^{\frac{\alpha}{1-\alpha}} x^{-\frac{1}{1-\alpha}} e^{-B(u)^{\frac{1}{1-\alpha}} \lambda^{\frac{\alpha}{1-\alpha}} x^{-\frac{\alpha}{1-\alpha}}-x} \mathrm{~d} u, \quad x \in \mathbb{R}^{+},
$$

which is the marginal density of the bivariate variable $(X, U)$ on $[0, \infty) \times[0, \pi]$ with density

$$
f(x, u)=\frac{\alpha e^{\lambda^{\alpha}}}{\pi(1-\alpha)} B(u)^{\frac{1}{1-\alpha}} \lambda^{\frac{\alpha}{1-\alpha}} x^{-\frac{1}{1-\alpha}} \exp \left(-B(u)^{\frac{1}{1-\alpha}} \lambda^{\frac{\alpha}{1-\alpha}} x^{-\frac{\alpha}{1-\alpha}}-x\right) .
$$

To sample $S_{\alpha, \lambda}$, first, we sample ( $\left.X, U\right)$ by applying the two-dimensional SR scheme in (2.1), and then return

$$
S_{\alpha, \lambda}=X / \lambda
$$

To simulate $(X, U)$ with density (3.2), we could choose a gamma-uniform bivariate envelope $\left(X^{\prime}, U^{\prime}\right)$ on $[0, \infty) \times[0, \pi]$ with density

$$
g(x, u)=\frac{1}{\pi} \frac{1}{\Gamma(m)} x^{m-1} e^{-x},
$$

for some $m \in \mathbb{R}^{+}$. Given the density function $f(x, u)$ for $(X, U)$ in (3.2) and $g(x, u)$ for $\left(X^{\prime}, U^{\prime}\right)$ in (3.3), we have

$$
\frac{f(x, u)}{g(x, u)}
$$




$$
\begin{aligned}
& =\frac{\alpha e^{\lambda^{\alpha}} \Gamma(m)}{1-\alpha} B(u)^{\frac{1}{1-\alpha}} \lambda^{\frac{\alpha}{1-\alpha}} x^{-\frac{\alpha}{1-\alpha}-m} \exp \left(-B(u)^{\frac{1}{1-\alpha}} \lambda^{\frac{\alpha}{1-\alpha}} x^{-\frac{\alpha}{1-\alpha}}\right) \\
& \leq \frac{\alpha e^{\lambda^{\alpha}} \Gamma(m)}{1-\alpha} B(u)^{\frac{1}{1-\alpha}} \lambda^{\frac{\alpha}{1-\alpha}}\left[\frac{\frac{\alpha}{1-\alpha} B(u)^{\frac{1}{1-\alpha}} \lambda^{\frac{\alpha}{1-\alpha}}}{\frac{\alpha}{1-\alpha}+m}\right]^{-\frac{(1-\alpha) m+\alpha}{\alpha}} \exp \left(-B(u)^{\frac{1}{1-\alpha}} \lambda^{\frac{\alpha}{1-\alpha}}\left[\frac{\alpha B(u)^{\frac{1}{1-\alpha}} \lambda^{\frac{\alpha}{1-\alpha}}}{\alpha+(1-\alpha) m}\right]^{-1}\right) \\
& =\left(\frac{\alpha}{1-\alpha}\right)^{-\frac{m(1-\alpha)}{\alpha}} \lambda^{-m} \Gamma(m)\left(\frac{\alpha}{1-\alpha}+m\right)^{\frac{m(1-\alpha)+\alpha}{\alpha}} e^{-\frac{m(1-\alpha)+\alpha}{\alpha}+\lambda^{\alpha}} B(u)^{-\frac{m}{\alpha}} \\
& \leq\left(\frac{\alpha}{1-\alpha}\right)^{-\frac{m(1-\alpha)}{\alpha}} \lambda^{-m} \Gamma(m)\left(\frac{\alpha}{1-\alpha}+m\right)^{\frac{m(1-\alpha)+\alpha}{\alpha}} e^{-\frac{m(1-\alpha)+\alpha}{\alpha}+\lambda^{\alpha}} B(0)^{-\frac{m}{\alpha}} \\
& =\left(\frac{\alpha}{1-\alpha}\right)^{-\frac{m(1-\alpha)}{\alpha}} \lambda^{-m} \Gamma(m)\left(\frac{\alpha}{1-\alpha}+m\right)^{\frac{m(1-\alpha)+\alpha}{\alpha}} e^{-\frac{m(1-\alpha)+\alpha}{\alpha}+\lambda^{\alpha}}\left[(1-\alpha)^{1-\alpha} \alpha^{\alpha}\right]^{-\frac{m}{\alpha}} \\
& =C_{1}(\alpha, \lambda ; m),
\end{aligned}
$$

where $B(u)$ is a monotone increasing function with

$$
\min _{0 \leq u \leq \infty}\{B(u)\}=B(0)=(1-\alpha)^{1-\alpha} \alpha^{\alpha}
$$

The A/R constant $C_{1}(\alpha, \lambda ; m)$ can be further minimised over $m$. The optimal value $m^{*}$ satisfies

$$
\frac{\alpha}{1-\alpha} \psi^{(0)}\left(m^{*}\right)+\ln \left(\frac{\alpha}{1-\alpha}+m^{*}\right)=\ln \left(\alpha^{\frac{1}{1-\alpha}} \lambda^{\frac{\alpha}{1-\alpha}}\right), \quad \text { for } \psi^{(0)}(m)=\frac{\mathrm{d} \Gamma(m)}{\mathrm{d} m}
$$

Hence, by approximating the LHS of (3.5), the optimal rate $m^{*}$ for the gamma-distributed envelope is chosen by setting $m^{*}=\alpha \lambda^{\alpha}$. The $\mathrm{A} / \mathrm{R}$ decision therefore follows

$$
V \leq \frac{f\left(X^{\prime}, U^{\prime}\right)}{C_{1}(\alpha, \lambda) g\left(X^{\prime}, U^{\prime}\right)}
$$

with

$$
C_{1}(\alpha, \lambda)=C_{1}\left(\alpha, \lambda ; \alpha \lambda^{\alpha}\right)=\left(\alpha \lambda^{\alpha}\right)^{-\lambda^{\alpha}} e^{\alpha \lambda^{\alpha}-1} \Gamma\left(\alpha \lambda^{\alpha}\right)\left(\frac{\alpha}{1-\alpha}+\alpha \lambda^{\alpha}\right)^{\lambda^{\alpha}(1-\alpha)+1}
$$

where $C_{1}(\alpha, \lambda)$ is the associated $\mathrm{A} / \mathrm{R}$ constant to sample $(X, U)$ via a gamma-uniform bivariate envelope $\left(X^{\prime}, U^{\prime}\right)$. Instead of this gamma-uniform bivariate envelope, one could use a gamma and truncated-normal bivariate envelope $(\bar{X}, \bar{U})$ on $[0, \infty) \times[0, \pi]$ with associated density of the form

$$
h(x, u)=\frac{x^{\alpha \lambda^{\alpha}-1} e^{-x}}{\Gamma\left(\alpha \lambda^{\alpha}\right)} \frac{\sqrt{2 \alpha(1-\alpha) \lambda^{\alpha}} / \sqrt{\pi}}{\operatorname{Erf}\left(\pi \sqrt{\alpha(1-\alpha) \lambda^{\alpha} / 2}\right)} e^{-\frac{\alpha(1-\alpha) \lambda^{\alpha} u^{2}}{2}}
$$

to implement the two-dimensional SR scheme. We consider a new envelope $(\bar{X}, \bar{U})$ such that

$$
\bar{X} \sim \Gamma\left(\alpha \lambda^{\alpha}, 1\right), \quad \bar{U} \sim \mathcal{N}\left(\mu=0, \sigma^{2}=\frac{1}{\alpha(1-\alpha) \lambda^{\alpha}}, \mathrm{lb}=0, \mathrm{ub}=\pi\right)
$$


which is a truncated-normal random variable with mean $\mu=0$ and variance $\sigma^{2}=\frac{1}{\alpha(1-\alpha) \lambda^{\alpha}}$ within the domain $[0, \pi]$. Given the joint density of $(X, U)$ in (3.2) and the joint density of $(\bar{X}, \bar{U})$ in (3.7), first, by maximising $f(x, u) / g(x, u)$ with respect to $x$, we have

$$
\begin{aligned}
\frac{f(x, u)}{h(x, u)} \leq & \frac{\operatorname{Erf}\left(\pi \sqrt{\alpha(1-\alpha) \lambda^{\alpha} / 2}\right) \alpha e^{\lambda^{\alpha}} \Gamma\left(\alpha \lambda^{\alpha}\right)}{(1-\alpha) \sqrt{2 \pi \alpha(1-\alpha) \lambda^{\alpha}}} \lambda^{-\alpha \lambda^{\alpha}} B(u)^{-\lambda^{\alpha}} e^{\frac{\alpha(1-\alpha) \lambda^{\alpha} u^{2}}{2}} \\
& \times\left(1+(1-\alpha) \lambda^{\alpha}\right)^{1+(1-\alpha) \lambda^{\alpha}} e^{-\left(1+(1-\alpha) \lambda^{\alpha}\right)} .
\end{aligned}
$$

According to Devroye (2009), we have the inequality

$$
B(u)^{-\lambda^{\alpha}} \leq B(0)^{-\lambda^{\alpha}} e^{-\frac{\alpha(1-\alpha) \lambda^{\alpha} u^{2}}{2}}=\left[\alpha^{\alpha}(1-\alpha)^{1-\alpha}\right]^{-\lambda^{\alpha}} e^{-\frac{\alpha(1-\alpha) \lambda^{\alpha} u^{2}}{2}}
$$

Hence, by (3.8), we then have

$$
\begin{aligned}
\frac{f(x, u)}{h(x, u)} & \leq \frac{\operatorname{Erf}\left(\pi \sqrt{\alpha(1-\alpha) \lambda^{\alpha} / 2}\right) \Gamma\left(\alpha \lambda^{\alpha}+1\right) e^{-1+\alpha \lambda^{\alpha}}\left(\alpha \lambda^{\alpha}\right)^{-\alpha \lambda^{\alpha}}(1-\alpha)^{-1-(1-\alpha) \lambda^{\alpha}}}{\sqrt{2 \pi \alpha(1-\alpha) \lambda^{\alpha}}\left(\frac{1}{\lambda^{\alpha}}+(1-\alpha)\right)^{-1-(1-\alpha) \lambda^{\alpha}}} \\
& \leq \frac{\Gamma\left(\alpha \lambda^{\alpha}+1\right) e^{-1+\alpha \lambda^{\alpha}}\left(\alpha \lambda^{\alpha}\right)^{-\alpha \lambda^{\alpha}}}{\sqrt{2 \pi \alpha(1-\alpha) \lambda^{\alpha}}}\left(1+\frac{1}{(1-\alpha) \lambda^{\alpha}}\right)^{1+(1-\alpha) \lambda^{\alpha}} \\
& =C_{3}(\alpha, \lambda),
\end{aligned}
$$

where $C_{3}(\alpha, \lambda)$ is the associated $\mathrm{A} / \mathrm{R}$ constant to sample $(X, U)$ via a gamma and truncated-normal bivariate envelope $(\bar{X}, \bar{U})$. Given these two methodologies, one could set $S_{\alpha, \lambda}=X / \lambda$ to obtain the realisation of $S_{\alpha, \lambda}$ once $X$ has been generated.

Besides $X=\lambda S_{\alpha, \lambda}$, we could use an alternative transformation to sample $S_{\alpha, \lambda}$ by setting

$$
Z=B(U)^{\frac{1}{1-\alpha}} S_{\alpha, \lambda}^{-\frac{\alpha}{1-\alpha}}
$$

According to (2.5), by changing the variables of the joint distribution function from $(S, U)$ to $(Z, U)$, the bivariate density function of $(Z, U)$ in $(z, u)$ on $[0, \infty) \times[0, \pi]$ is of the form

$$
f(z, u)=\frac{e^{\lambda^{\alpha}}}{\pi} \exp \left(-z-\lambda B(u)^{\frac{1}{\alpha}} z^{-\frac{1-\alpha}{\alpha}}\right)
$$

To sample $S_{\alpha, \lambda}$, we could sample $(Z, U)$ first, and then return

$$
S_{\alpha, \lambda}=B(U)^{\frac{1}{\alpha}} Z^{-\frac{1-\alpha}{\alpha}}
$$

To simulate $(Z, U)$ with density (3.11), we choose an envelope $\left(Z^{\prime}, U^{\prime}\right)$ on $[0, \infty) \times[0, \pi]$ with 
joint density function

$$
g(z, u)=\frac{1}{\pi} \frac{z^{r} e^{-z}}{\Gamma(r+1)}
$$

According to (3.4), we have

$$
\begin{aligned}
\frac{f(z, u)}{g(z, u)} & \leq e^{\lambda^{\alpha}} \Gamma(r+1) z^{-r} \exp \left(-\lambda \alpha(1-\alpha)^{\frac{1-\alpha}{\alpha}} z^{-\frac{1-\alpha}{\alpha}}\right) \\
& \leq\left(\frac{\alpha r}{(1-\alpha) \lambda}\right)^{\frac{r \alpha}{1-\alpha}} e^{-\frac{r \alpha}{1-\alpha}+\lambda^{\alpha}} \Gamma(r+1)\left[(1-\alpha) \alpha^{\frac{\alpha}{1-\alpha}}\right]^{-r}=C_{2}(\alpha, \lambda ; r),
\end{aligned}
$$

where $C_{2}(\alpha, \lambda ; r)$ can be minimised over $r$. The optimal value $r^{*}$ satisfies

$$
\psi^{(0)}\left(r^{*}+1\right)=\frac{\alpha}{1-\alpha} \ln \left(\frac{\lambda(1-\alpha)^{\frac{1}{\alpha}}}{r^{*}}\right), \quad \text { for } \psi^{(0)}(r)=\frac{\mathrm{d} \Gamma(r)}{\mathrm{d} r} .
$$

By approximating the LHS of (3.12), the optimal rate $r^{*}$ is chosen by setting $r^{*}=(1-\alpha) \lambda^{\alpha}$. Hence, the associated $\mathrm{A} / \mathrm{R}$ constant with $r^{*}$ is given by

$$
C_{2}(\alpha, \lambda)=C_{2}\left(\alpha, \lambda ;(1-\alpha) \lambda^{\alpha}\right)=\Gamma\left((1-\alpha) \lambda^{\alpha}+1\right) e^{(1-\alpha) \lambda^{\alpha}}\left((1-\alpha) \lambda^{\alpha}\right)^{-(1-\alpha) \lambda^{\alpha}},
$$

where $C_{2}(\alpha, \lambda)$ is the associated $\mathrm{A} / \mathrm{R}$ constant to sample $(Z, U)$ via a gamma-uniform bivariate envelope $\left(Z^{\prime}, U^{\prime}\right)$. Similarly, one could also consider a gamma and truncated-normal bivariate envelope $(\bar{Z}, \bar{U})$ for $(Z, U)$ on $[0, \infty) \times[0, \pi]$ with density (3.11), such that

$$
\bar{Z} \sim \Gamma\left((1-\alpha) \lambda^{\alpha}+1,1\right), \quad \bar{U} \sim \mathcal{N}\left(\mu=0, \sigma^{2}=\frac{1}{\alpha(1-\alpha) \lambda^{\alpha}}, \mathrm{lb}=0, \mathrm{ub}=\pi\right)
$$

The joint density is given as

$$
h(z, u)=\frac{z^{(1-\alpha) \lambda^{\alpha}} e^{-z}}{\Gamma\left((1-\alpha) \lambda^{\alpha}+1\right)} \frac{\sqrt{2 \alpha(1-\alpha) \lambda^{\alpha}} / \sqrt{\pi}}{\operatorname{Erf}\left(\pi \sqrt{\alpha(1-\alpha) \lambda^{\alpha} / 2}\right)} e^{-\frac{\alpha(1-\alpha) \lambda^{\alpha} u^{2}}{2}}
$$

Then, by maximising $f(z, u) / h(z, u)$ with respect to $z$ and applying inequality (3.8), we have

$$
\begin{aligned}
\frac{f(z, u)}{h(z, u)} & =\frac{\operatorname{Erf}\left(\pi \sqrt{\alpha(1-\alpha) \lambda^{\alpha} / 2}\right) e^{\lambda^{\alpha}} \Gamma\left((1-\alpha) \lambda^{\alpha}+1\right)}{\sqrt{2 \pi \alpha(1-\alpha) \lambda^{\alpha}}} z^{-(1-\alpha) \lambda^{\alpha}} \exp \left(-\lambda B(u)^{\frac{1}{\alpha}} z^{-\frac{1-\alpha}{\alpha}}+\frac{\alpha(1-\alpha) \lambda^{\alpha} u^{2}}{2}\right) \\
& \leq \frac{\operatorname{Erf}\left(\pi \sqrt{\alpha(1-\alpha) \lambda^{\alpha} / 2}\right) e^{(1-\alpha) \lambda^{\alpha}} \Gamma\left((1-\alpha) \lambda^{\alpha}+1\right)}{\sqrt{2 \pi \alpha(1-\alpha) \lambda^{\alpha}}} \lambda^{-\alpha(1-\alpha) \lambda^{\alpha}} \alpha^{\alpha \lambda^{\alpha}} B(u)^{-\lambda^{\alpha}} e^{\frac{\alpha(1-\alpha) \lambda^{\alpha} u^{2}}{2}} \\
& \leq \frac{\operatorname{Erf}\left(\pi \sqrt{\alpha(1-\alpha) \lambda^{\alpha} / 2}\right) e^{(1-\alpha) \lambda^{\alpha}} \Gamma\left((1-\alpha) \lambda^{\alpha}+1\right)}{\sqrt{2 \pi \alpha(1-\alpha) \lambda^{\alpha}}}(1-\alpha)^{-(1-\alpha) \lambda^{\alpha}} \lambda^{-\alpha(1-\alpha) \lambda^{\alpha}} \\
& \leq \frac{\Gamma\left((1-\alpha) \lambda^{\alpha}+1\right)}{\sqrt{2 \pi \alpha(1-\alpha) \lambda^{\alpha}}}\left((1-\alpha) \lambda^{\alpha}\right)^{-(1-\alpha) \lambda^{\alpha}} e^{(1-\alpha) \lambda^{\alpha}}=C_{4}(\alpha, \lambda)
\end{aligned}
$$


where $C_{4}(\alpha, \lambda)$ is the associated $\mathrm{A} / \mathrm{R}$ constant to sample $(Z, U)$ via a gamma and truncated-normal bivariate envelope $(\bar{Z}, \bar{U})$. Given these two methodologies, one could set $S_{\alpha, \lambda}=B(U)^{\frac{1}{\alpha}} Z^{-\frac{1-\alpha}{\alpha}}$ to obtain the realisation of $S_{\alpha, \lambda}$ once $X$ has been generated.

When specifying the input of $(\alpha, \lambda)$, to sample $S_{\alpha, \lambda}$, these four two-dimensional SR schemes will lead to different expected complexities, namely $C_{1}(\alpha, \lambda), C_{2}(\alpha, \lambda), C_{3}(\alpha, \lambda), C_{4}(\alpha, \lambda)$. Therefore, the most efficient strategy to sample $S_{\alpha, \lambda}$ is to choose the one with the smallest highest acceptance rate, to implement the corresponding two-dimensional single rejection procedure, which leads to Algorithm 3.1. And the overall complexity therefore would be formidable by $C(\alpha, \lambda)$, where

$$
C(\alpha, \lambda)=\min _{i=1,2,3,4}\left\{C_{i}(\alpha, \lambda):(\alpha, \lambda) \in(0,1) \times(0, \infty)\right\}
$$

Given the complexity $C(\alpha, \lambda)$ in (3.15) for Algorithm 3.1, we conclude the following result.

Theorem 3.1. The complexity $C(\alpha, \lambda)$ in (3.15) for Algorithm 3.1 is uniformly bounded. In particular, we have

$$
\sup _{\alpha \in(0,1), \lambda \geq 0} C(\alpha, \lambda) \leq 4.2154
$$

Proof. According to Corollary 1.2 in Batir (2008), the following inequality

$$
\Gamma(x+1)<\sqrt{2 \pi} x^{x} e \sqrt{x+\frac{1}{2}}
$$

hold for $x \geq 0$, we then have the following results

$$
\begin{aligned}
C_{1}(\alpha, \lambda) & <\sqrt{2 \pi \alpha \lambda^{\alpha}+\pi}\left(1+\frac{1}{(1-\alpha) \lambda^{\alpha}}\right)=\bar{C}_{1}(\alpha, \lambda), \\
C_{2}(\alpha, \lambda) & <\sqrt{2 \pi(1-\alpha) \lambda^{\alpha}+\pi}=\bar{C}_{2}(\alpha, \lambda), \\
C_{3}(\alpha, \lambda) & <\sqrt{\frac{1}{1-\alpha}+\frac{1}{2 \alpha(1-\alpha) \lambda^{\alpha}}}\left(1+\frac{1}{(1-\alpha) \lambda^{\alpha}}\right)=\bar{C}_{3}(\alpha, \lambda), \\
C_{4}(\alpha, \lambda) & <\sqrt{\frac{1}{\alpha}+\frac{1}{2 \alpha(1-\alpha) \lambda^{\alpha}}}=\bar{C}_{4}(\alpha, \lambda) .
\end{aligned}
$$

Hence, for any combination of $(\alpha, \lambda) \in(0,1) \times(0, \infty)$, we have

$$
\begin{aligned}
C(\alpha, \lambda) & =\min _{i=1,2,3,4}\left\{C_{i}(\alpha, \lambda):(\alpha, \lambda) \in(0,1) \times(0, \infty)\right\} \\
& <\min _{i=1,2,3,4}\left\{\bar{C}_{i}(\alpha, \lambda):(\alpha, \lambda) \in(0,1) \times(0, \infty)\right\} \\
& =\bar{C}(\alpha, \lambda) .
\end{aligned}
$$


To prove $C(\alpha, \lambda)$ is uniformly bounded over $(\alpha, \lambda) \in(0,1) \times(0, \infty)$, it suffices to prove $\bar{C}(\alpha, \lambda)$ is uniformly bounded over $(\alpha, \lambda) \in(0,1) \times(0, \infty)$. We notice that the following inequalities hold

$$
\begin{aligned}
\min \left\{\sqrt{2 \pi \alpha \lambda^{\alpha}+\pi}, \sqrt{\frac{1}{1-\alpha}+\frac{1}{2 \alpha(1-\alpha) \lambda^{\alpha}}}\right\} & \leq \sqrt{\frac{1}{1-\alpha}+\pi}, \\
\min \left\{\sqrt{2 \pi(1-\alpha) \lambda^{\alpha}+\pi}, \sqrt{\frac{1}{\alpha}+\frac{1}{2 \alpha(1-\alpha) \lambda^{\alpha}}}\right\} & \leq \sqrt{\frac{1}{\alpha}+\pi},
\end{aligned}
$$

for any arbitrary $(\alpha, \lambda) \in(0,1) \times(0, \infty)^{4}$, which indicate that

$$
\begin{aligned}
\min \left\{\bar{C}_{1}(\alpha, \lambda), \bar{C}_{3}(\alpha, \lambda)\right\} & \leq \sqrt{\frac{1}{1-\alpha}+\pi}\left(1+\frac{1}{(1-\alpha) \lambda^{\alpha}}\right) \\
\min \left\{\bar{C}_{2}(\alpha, \lambda), \bar{C}_{4}(\alpha, \lambda)\right\} & \leq \sqrt{\frac{1}{\alpha}+\pi}
\end{aligned}
$$

Hence, we have

$$
\bar{C}(\alpha, \lambda) \leq \min \left\{\bar{C}_{2}(\alpha, \lambda), \sqrt{\frac{1}{\alpha}+\pi}, \sqrt{\frac{1}{1-\alpha}+\pi}\left(1+\frac{1}{(1-\alpha) \lambda^{\alpha}}\right)\right\}
$$

Note that, since $\bar{C}_{2}(\alpha, \lambda)$ goes to $\sqrt{3 \pi}$ when both $\alpha, \lambda \rightarrow 0$, this $\bar{C}_{2}(\alpha, \lambda)$ in (3.17) will prevent explosion when both $\alpha, \lambda \rightarrow 0$.

First, for the case $\alpha \in\left[\frac{1}{2}, 1\right)$, since $\sqrt{\frac{1}{\alpha}+\pi}$ is decreasing and bounded, we have

$$
\bar{C}(\alpha, \lambda) \leq \sqrt{\frac{1}{\alpha}+\pi} \leq \sqrt{2+\pi} \approx 2.2675
$$

For the case $\alpha \in\left(0, \frac{1}{2}\right)$, we have

$$
\begin{aligned}
\bar{C}(\alpha, \lambda) & \leq \min \left\{\sqrt{2 \pi(1-\alpha) \lambda^{\alpha}+\pi}, \sqrt{\frac{1}{1-\alpha}+\pi}\left(1+\frac{1}{(1-\alpha) \lambda^{\alpha}}\right)\right\} \\
& \leq \min \left\{\sqrt{2 \pi \lambda^{\alpha}+\pi}, \sqrt{2+\pi}\left(1+\frac{2}{\lambda^{\alpha}}\right)\right\} \\
& \leq \max _{(\alpha, \lambda) \in(0,1) \times(0, \infty)}\left\{\min \left\{\sqrt{2 \pi \lambda^{\alpha}+\pi}, \sqrt{2+\pi}\left(1+\frac{2}{\lambda^{\alpha}}\right)\right\}\right\} \approx 4.2154,
\end{aligned}
$$

where this supreme value is obtained at $\lambda^{\alpha} \approx 2.3281$, i.e. when

$$
\sqrt{2 \pi \lambda^{\alpha}+\pi}=\sqrt{2+\pi}\left(1+\frac{2}{\lambda^{\alpha}}\right)
$$

This is because one of the function is increasing in $\lambda^{\alpha}$ and the other function is decreasing in $\lambda^{\alpha}$, the maximum of the minimum of these two function over $(\alpha, \lambda) \in(0,1) \times(0, \infty)$ is at the point

\footnotetext{
${ }^{4}$ The supreme value of the function defined by the minimum of an increasing and decreasing function is at the point when the two functions meet.
} 
when these two functions are equal.

Hence, by (3.16), we have

$$
C(\alpha, \lambda) \leq \bar{C}(\alpha, \lambda) \leq 4.2154 \times \mathbf{1}_{\{0<\alpha<1 / 2\}}+2.2675 \times \mathbf{1}_{\{1 / 2 \leq \alpha<1\}} \leq 4.2154,
$$

which clearly implies that $C(\alpha, \lambda)$ is uniformly bounded by 4.2154 over $(0,1) \times(0, \infty)$.

This $C(\alpha, \lambda)$ is uniformly bounded by 4.2154 over all combinations of the parameters. When the stability parameter $\alpha$ is between $1 / 2$ and 1 , the upper bound can be reduced to 2.2685 . In principle, the uniform bound provided in Theorem 3.1 is the bound for $\bar{C}(\alpha, \lambda)$, which is the upper bound of $C(\alpha, \lambda)$, whereas the actual bound of $C(\alpha, \lambda)$ is much smaller than this uniform bound.

Remark 3.1. Given $C(\alpha, \lambda)$ in (3.15), when holding $\lambda$ fixed, we have

$$
\begin{aligned}
& \lim _{\alpha \rightarrow 0} C(\alpha, \lambda)=\lim _{\alpha \rightarrow 0} C_{1}(\alpha, \lambda)=\lim _{\alpha \rightarrow 0} e^{-1}\left(1+\frac{1}{(1-\alpha) \lambda^{\alpha}}\right)^{\lambda^{\alpha}+1}=\frac{4}{e}, \\
& \lim _{\alpha \rightarrow 1} C(\alpha, \lambda)=\lim _{\alpha \rightarrow 1} C_{2}(\alpha, \lambda)=\lim _{x \rightarrow 0} \Gamma(x+1) x^{-x} e^{x}=1,
\end{aligned}
$$

and

$$
\begin{aligned}
\lim _{\lambda \rightarrow 0} C(\alpha, \lambda) & =\lim _{\lambda \rightarrow 0} C_{2}(\alpha, \lambda)=\lim _{x \rightarrow 0} \Gamma(x+1) x^{-x} e^{x}=1, \\
\lim _{\lambda \rightarrow \infty} C(\alpha, \lambda) & \leq \lim _{\lambda \rightarrow \infty} \bar{C}(\alpha, \lambda)=\min _{\alpha \in(0,1)}\{\sqrt{1 / \alpha}, \sqrt{1 / 1-\alpha}\} \leq \sqrt{2},
\end{aligned}
$$

while holding $\alpha$ fixed. Figure 2 shows the value of $C(\alpha, \lambda)$ for various values of $\alpha$ and $\lambda$. The calculated maximum it attains for those values is about 2.5. This actual bound for $C(\alpha, \lambda)$ we observed is much smaller than the one we discovered in Theorem 3.1.

In fact, this $C(\alpha, \lambda)$ is indeed the complexity of the scheme that optimally combines the four two-dimensional SR algorithms with different envelopes and implements the most efficient algorithm by choosing the one with the smallest $\mathrm{A} / \mathrm{R}$ constant to sample the exponential tilted stable random variable $S_{\alpha, \lambda}$. The overall complexity of Algorithm 3.1 is $C(\alpha, \lambda)$ in (3.15), which, according to Theorem 3.1, is uniformly bounded by 4.2154 . Apparently, the complexity is smaller than 8.1133, which is the complexity of the DR scheme (Devroye, 2009), and the relevant numerical comparison tests of these algorithms will be illustrated in Section 5. Figure 1 represents the plot of the regions over $(0,1) \times(0, \infty)$ where each of the two-dimensional SR algorithms suggested in Algorithm 3.1 will be active. We can see that for $\alpha$ close to 0, the two-dimensional SR 


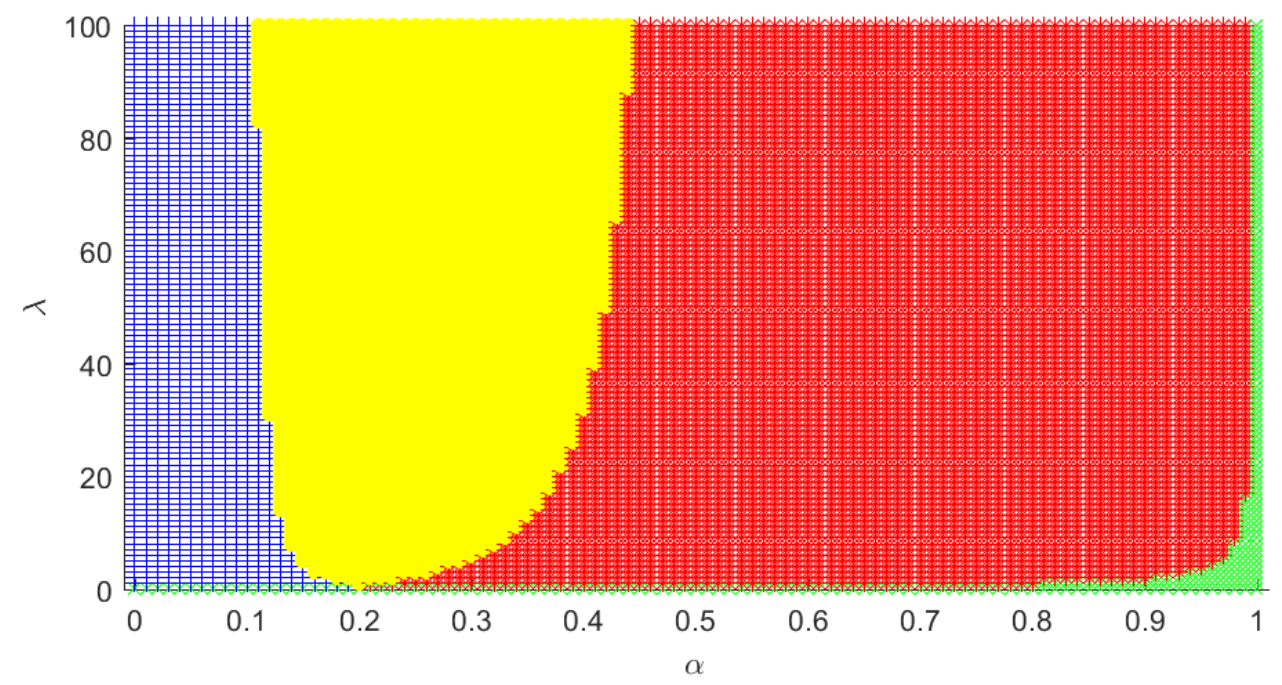

Figure 1: Algorithm active regions: blue region represents $C(\alpha, \lambda)=C_{1}(\alpha, \lambda)$; green region represents $C(\alpha, \lambda)=C_{2}(\alpha, \lambda)$; yellow region represents $C(\alpha, \lambda)=C_{3}(\alpha, \lambda)$; red region represents $C(\alpha, \lambda)=C_{4}(\alpha, \lambda)$.

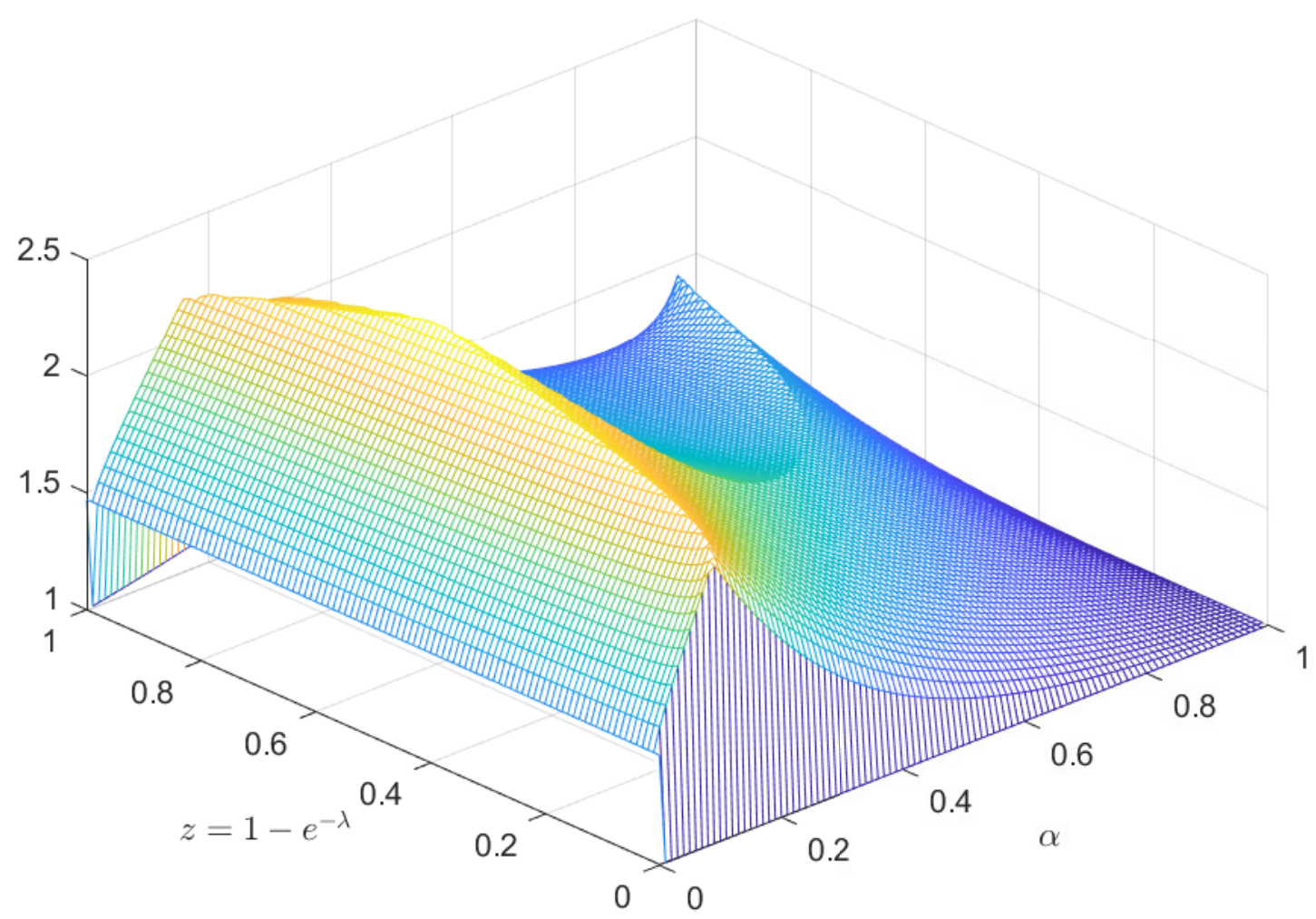

Figure 2: The complexity of Algorithm 3.1 for $\alpha \in(0,1)$ and $\lambda:=-\ln (1-z)$ with $z \in(0,1)$ 
algorithm with $C_{1}(\alpha, \lambda)$ will be active and for $\alpha$ close to 1 , or $\lambda$ close to 0 , the two-dimensional SR algorithm with $C_{3}(\alpha, \lambda)$ will be active. When $\lambda$ getting large, depending on the size of $\alpha$, one of the two-dimensional SR algorithms with $C_{2}(\alpha, \lambda)$ and $C_{4}(\alpha, \lambda)$ will be active. The limits for $C(\alpha, \lambda)$ provided in Remark 3.1 clearly explain these facts.

\section{Simulation for Gamma Tilted Stable Distribution}

Beside the ETS class, the gamma tilted stable (GTS) distribution is another interesting class of tilted stable distributions. The GTS, denoted by $G_{\alpha, \lambda, \nu}$, was first introduced by Barndorff-Nielsen and Shephard (2001), and its density is defined as

$$
f(s)=\frac{s^{\nu} e^{-\lambda s} f_{\alpha}(s)}{G(\nu, \lambda)}, \quad s \in \mathbb{R}^{+}
$$

where $\nu>0$, and $G(\nu, \lambda)=\int_{0}^{\infty} y^{\nu} e^{-\lambda y} f_{\alpha}(y) \mathrm{d} y$, and $f_{\alpha}(\cdot)$ is the density of the positive stable variable $S_{\alpha}$ in (2.2). Based on the two-dimensional single rejection method and the gamma-uniform envelope and the gamma and truncated-normal envelope for the ETS, we can also develop a simulation scheme to sample the GTS variables. The details are provided in Algorithm 4.1.

Algorithm 4.1. We have the following simulation scheme for the gamma tilted stable $G_{\alpha, \lambda, \nu}$,

1. set $R_{1}=\operatorname{Erf}\left(\sqrt{\alpha(1-\alpha) \lambda^{\alpha} \pi^{2} / 2}\right), R_{2}=\operatorname{Erf}\left(\pi \sqrt{(1-\alpha)\left(\alpha \lambda^{\alpha}-\nu\right) / 2}\right)$

2. set $G_{1}=\frac{e^{\alpha \lambda^{\alpha}-1}}{\left(\alpha \lambda^{\alpha}\right)^{\lambda^{\alpha}}}\left(\frac{\alpha+\alpha(1-\alpha) \lambda^{\alpha}}{1-\alpha}\right)^{\lambda^{\alpha}(1-\alpha)+1}, G_{2}=\frac{e^{(1-\alpha) \lambda^{\alpha}(1-\alpha)} \frac{(1-\alpha) \nu}{\alpha} \alpha^{\nu}}{(1-a)^{(1-\alpha) \lambda^{\alpha}} \lambda^{\alpha(1-\alpha) \lambda^{\alpha}}}$,

$G_{3}=\frac{\lambda^{-\alpha^{2} \lambda^{\alpha}+\alpha} e^{-1+\alpha \lambda^{\alpha}} \alpha^{-\alpha \lambda^{\alpha}+1}(1-\alpha)^{-1-(1-\alpha) \lambda^{\alpha}}}{\sqrt{2 \pi \alpha(1-\alpha) \lambda^{\alpha}}\left(1 / \lambda^{\alpha}+(1-\alpha)\right)^{-1-(1-\alpha) \lambda^{\alpha}}}, G_{4}=\frac{e^{(1-\alpha) \lambda^{\alpha}}(1-\alpha)^{\frac{(1-\alpha) \nu}{\alpha}} \alpha^{\nu}}{\sqrt{2 \pi \alpha(1-\alpha) \lambda^{\alpha}} \sqrt{1-\nu /\left[\alpha \lambda^{\alpha}\right]}\left((1-\alpha) \lambda^{\alpha}\right)^{(1-\alpha) \lambda^{\alpha}}}$, $\bar{C}_{1}=\Gamma\left(\alpha \lambda^{\alpha}+\nu\right) \lambda^{-\nu} G_{1}, \bar{C}_{3}=\Gamma\left(\alpha \lambda^{\alpha}+\nu\right) \lambda^{-\nu} G_{3}, \bar{C}_{2}=\Gamma\left((1-\alpha) \lambda^{\alpha}-(1-\alpha) \nu / \alpha+1\right) G_{2}$, and $\bar{C}_{4}=\Gamma\left((1-\alpha) \lambda^{\alpha}-(1-\alpha) \nu / \alpha+1\right) G_{4}$

3. if $\left(\left\{\bar{C}_{1}=\min \left\{\bar{C}_{1}, \bar{C}_{2}, \bar{C}_{3}, \bar{C}_{4}\right\}, \nu<\alpha \lambda^{\alpha}\right\}\right.$ or $\left.\left\{\bar{C}_{1}=\min \left\{\bar{C}_{1}, \bar{C}_{3}\right\}, \nu \geq \alpha \lambda^{\alpha}\right\}\right)\{$

4. repeat \{

5. $\quad$ sample $U \sim \mathcal{U}[0, \pi], X \sim \Gamma\left(\alpha \lambda^{\alpha}+\nu, 1\right), V \sim \mathcal{U}[0,1] ;$ set $G=X / \lambda$

6. $\quad$ if $\left(V \leq \frac{\alpha e^{\lambda^{\alpha}}}{G_{1}(1-\alpha)} B(U)^{\frac{1}{1-\alpha}} \lambda^{\frac{\alpha}{1-\alpha}} X^{-\frac{\alpha}{1-\alpha}-\alpha \lambda^{\alpha}} e^{-B(U)^{\frac{1}{1-\alpha}} \lambda^{\frac{\alpha}{1-\alpha}} X^{-\frac{\alpha}{1-\alpha}}}\right)$ break

7. $\}$

8. $\}$ 
9. if $\left(\bar{C}_{2}=\min \left\{\bar{C}_{1}, \bar{C}_{2}, \bar{C}_{3}, \bar{C}_{4}\right\}, \nu<\alpha \lambda^{\alpha}\right)\{$

10. repeat \{

11. sample $U \sim \mathcal{U}(0, \pi)$

12. $\quad$ sample $Z \sim \Gamma\left((1-\alpha) \lambda^{\alpha}-(1-\alpha) \nu / \alpha+1,1\right), V \sim \mathcal{U}[0,1]$; set $G=B(U)^{\frac{1}{\alpha}} Z^{-\frac{1-\alpha}{\alpha}}$

13. $\quad$ if $\left(V \leq e^{\lambda^{\alpha}} B(U)^{\frac{\nu}{\alpha}} Z^{-(1-\alpha) \lambda^{\alpha}} e^{-\lambda B(U)^{\frac{1}{\alpha}} Z^{-\frac{1-\alpha}{\alpha}}} / G_{2}\right)$ break

14. \}

15. $\}$

16. if $\left(\bar{C}_{3}=\min \left\{\bar{C}_{1}, \bar{C}_{2}, \bar{C}_{3}, \bar{C}_{4}\right\}, \nu<\alpha \lambda^{\alpha}\right\}$ or $\left.\left\{\bar{C}_{3}=\min \left\{\bar{C}_{1}, \bar{C}_{3}\right\}, \nu \geq \alpha \lambda^{\alpha}\right\}\right)\{$

17. repeat \{

18. $\quad$ sample $U \sim \mathcal{N}\left(\mu=0, \sigma^{2}=\left[\alpha(1-\alpha) \lambda^{\alpha}\right]^{-1}, l b=0, u b=\pi\right)$

19. $\quad$ sample $X \sim \Gamma\left(\alpha \lambda^{\alpha}+\nu, 1\right), V \sim \mathcal{U}[0,1]$; set $G=X / \lambda$

20. $\quad$ if $\left(V \leq \frac{R_{1} \alpha e^{\lambda^{\alpha}} \lambda^{\frac{\alpha}{1-\alpha}} B(U)^{\frac{1}{1-\alpha}}}{G_{3}(1-\alpha) \sqrt{2 \pi \alpha(1-\alpha) \lambda^{\alpha}} X^{\frac{\alpha}{1-\alpha}+\alpha \lambda^{\alpha}}} e^{-\left(\lambda B(u)^{\frac{1}{\alpha}} X^{-1}\right)^{\frac{\alpha}{1-\alpha}}+\frac{\alpha(1-\alpha) \lambda^{\alpha} U^{2}}{2}}\right)$ break

21. $\}$

22. $\}$

23. if $\left(\bar{C}_{4}=\min \left\{\bar{C}_{1}, \bar{C}_{2}, \bar{C}_{3}, \bar{C}_{4}\right\}, \nu<\alpha \lambda^{\alpha}\right)\{$

24. repeat

25. $\quad$ sample $U \sim \mathcal{N}\left(\mu=0, \sigma^{2}=\left[(1-\alpha)\left(\alpha \lambda^{\alpha}-\nu\right)\right]^{-1}, l b=0, u b=\pi\right)$

26. $\quad$ sample $Z \sim \Gamma\left((1-\alpha) \lambda^{\alpha}-(1-\alpha) \nu / \alpha+1,1\right), V \sim \mathcal{U}[0,1]$; set $G=B(U)^{\frac{1}{\alpha}} Z^{-\frac{1-\alpha}{\alpha}}$

27. $\quad$ if $\left(V \leq \frac{R_{2}}{G_{4} \sqrt{2 \pi(1-\alpha)\left(\alpha \lambda^{\alpha}-\nu\right)} Z^{(1-\alpha) \lambda^{\alpha}}} e^{\lambda^{\alpha}} B(U)^{\frac{\nu}{\alpha}} e^{-\lambda B(U)^{\frac{1}{\alpha}} Z^{-\frac{1-\alpha}{\alpha}}+\frac{(1-\alpha)\left(\alpha \lambda^{\alpha}-\nu\right) U^{2}}{2}}\right)$ break

28. $\}$

29. $\}$

30. return $G$

Proof. Given $X=\lambda G_{\alpha, \lambda, \nu}$, the bivariate density of $(X, U)$ on $[0, \infty) \times[0, \pi]$ is given by

$$
f(x, u)=\frac{\alpha \lambda^{-\nu} e^{\lambda^{\alpha}}}{G(\nu, \lambda) \pi(1-\alpha)} B(u)^{\frac{1}{1-\alpha}} \lambda^{\frac{\alpha}{1-\alpha}} x^{-\frac{1}{1-\alpha}+\nu} \exp \left(-B(u)^{\frac{1}{1-\alpha}} \lambda^{\frac{\alpha}{1-\alpha}} x^{-\frac{\alpha}{1-\alpha}}-x\right)
$$


To generate $(X, U)$, we consider a bivariate envelope $(\tilde{X}, \tilde{U})$ on $[0, \infty) \times[0, \pi]$ with $\tilde{X} \sim \Gamma\left(\alpha \lambda^{\alpha}+\nu, 1\right)$ and $\tilde{U} \sim \mathcal{U}[0, \pi]$, the joint density is given as

$$
p(x, u)=\frac{1}{\pi} \frac{x^{\alpha \lambda^{\alpha}+\nu-1} e^{-x}}{\Gamma\left(\alpha \lambda^{\alpha}+\nu\right)},
$$

hence, we have

$$
\begin{aligned}
\frac{f(x, u)}{p(x, u)} & =\frac{\lambda^{-\nu} \Gamma\left(\alpha \lambda^{\alpha}+\nu\right)}{G(\nu, \lambda)} \frac{\alpha e^{\lambda^{\alpha}}}{1-\alpha} B(u)^{\frac{1}{1-\alpha}} \lambda^{\frac{\alpha}{1-\alpha}} x^{-\alpha \lambda^{\alpha}-\frac{\alpha}{1-\alpha}} \exp \left(-B(u)^{\frac{1}{1-\alpha}} \lambda^{\frac{\alpha}{1-\alpha}} x^{-\frac{\alpha}{1-\alpha}}\right) \\
& =\frac{\lambda^{-\nu} \Gamma\left(\alpha \lambda^{\alpha}+\nu\right)}{G(\nu, \lambda) \Gamma\left(\alpha \lambda^{\alpha}\right)} \frac{\alpha e^{\lambda^{\alpha}} \Gamma\left(\alpha \lambda^{\alpha}\right)}{1-\alpha} B(u)^{\frac{1}{1-\alpha}} \lambda^{\frac{\alpha}{1-\alpha}} x^{-\alpha \lambda^{\alpha}-\frac{\alpha}{1-\alpha}} \exp \left(-B(u)^{\frac{1}{1-\alpha}} \lambda^{\frac{\alpha}{1-\alpha}} x^{-\frac{\alpha}{1-\alpha}}\right) \\
& \leq \frac{C_{1}(\alpha, \lambda) \lambda^{-\nu} \Gamma\left(\alpha \lambda^{\alpha}+\nu\right)}{G(\nu, \lambda) \Gamma\left(\alpha \lambda^{\alpha}\right)}=\bar{C}_{1},
\end{aligned}
$$

where $C_{1}(\alpha, \lambda)$ is defined in (3.6).

Alternatively, we consider a bivariate envelope $(\hat{X}, \hat{U})$ on $[0, \infty) \times[0, \pi]$ such that

$$
\hat{X} \sim \Gamma\left(\alpha \lambda^{\alpha}+\nu, 1\right), \quad \hat{U} \sim \mathcal{N}\left(\mu=0, \sigma^{2}=\frac{1}{\alpha(1-\alpha) \lambda^{\alpha}}, \mathrm{lb}=0, \mathrm{ub}=\pi\right)
$$

and the joint density $(\hat{X}, \hat{U})$ is given

$$
q(x, u)=\frac{x^{\alpha \lambda^{\alpha}+\nu-1} e^{-x}}{\Gamma\left(\alpha \lambda^{\alpha}+\nu\right)} \frac{\sqrt{2 \alpha(1-\alpha) \lambda^{\alpha}} / \sqrt{\pi}}{\operatorname{Erf}\left(\pi \sqrt{\alpha(1-\alpha) \lambda^{\alpha} / 2}\right)} e^{-\frac{\alpha(1-\alpha) \lambda^{\alpha} u^{2}}{2}} .
$$

According to the inequality (3.9), we have

$$
\begin{aligned}
\frac{f(x, u)}{q(x, u)}= & \frac{\lambda^{-\nu} \Gamma\left(\alpha \lambda^{\alpha}+\nu\right) \operatorname{Erf}\left(\pi \sqrt{\alpha(1-\alpha) \lambda^{\alpha} / 2}\right) \alpha e^{\lambda^{\alpha}}}{G(\nu, \lambda)(1-\alpha) \sqrt{2 \pi \alpha(1-\alpha) \lambda^{\alpha}}} B(u)^{\frac{1}{1-\alpha}} \lambda^{\frac{\alpha}{1-\alpha}} x^{-\frac{\alpha}{1-\alpha}-\alpha \lambda^{\alpha}} \\
& \times \exp \left(-B(u)^{\frac{1}{1-\alpha}} \lambda^{\frac{\alpha}{1-\alpha}} x^{-\frac{\alpha}{1-\alpha}}+\frac{\alpha(1-\alpha) \lambda^{\alpha} u^{2}}{2}\right) \\
= & \frac{\lambda^{-\nu} \Gamma\left(\alpha \lambda^{\alpha}+\nu\right)}{G(\nu, \lambda) \Gamma\left(\alpha \lambda^{\alpha}\right)} \frac{\operatorname{Erf}\left(\pi \sqrt{\alpha(1-\alpha) \lambda^{\alpha} / 2}\right) \alpha e^{\lambda^{\alpha}} \Gamma\left(\alpha \lambda^{\alpha}\right)}{(1-\alpha) \sqrt{2 \pi \alpha(1-\alpha) \lambda^{\alpha}}} B(u)^{\frac{1}{1-\alpha}} \lambda^{\frac{\alpha}{1-\alpha}} x^{-\frac{\alpha}{1-\alpha}-\alpha \lambda^{\alpha}} \\
& \times \exp \left(-B(u)^{\frac{1}{1-\alpha}} \lambda^{\frac{\alpha}{1-\alpha}} x^{-\frac{\alpha}{1-\alpha}}+\frac{\alpha(1-\alpha) \lambda^{\alpha} u^{2}}{2}\right) \\
\leq & \frac{C_{3}(\alpha, \lambda) \lambda^{-\nu} \Gamma\left(\alpha \lambda^{\alpha}+\nu\right)}{G(\nu, \lambda) \Gamma\left(\alpha \lambda^{\alpha}\right)}=\bar{C}_{3},
\end{aligned}
$$

which is the associated $\mathrm{A} / \mathrm{R}$ constant. 
For $\nu<\alpha \lambda^{\alpha}$, if we set

$$
Z=B(U)^{\frac{1}{1-\alpha}} \lambda^{\frac{\alpha}{1-\alpha}} X^{-\frac{\alpha}{1-\alpha}}
$$

then, the joint density of $(Z, U)$ on $[0, \infty) \times[0, \pi]$ is

$$
f(z, u)=\frac{e^{\lambda^{\alpha}}}{G(\nu, \lambda) \pi} B(u)^{\frac{\nu}{\alpha}} z^{-\frac{(1-\alpha) \nu}{\alpha}} \exp \left(-z-\lambda B(u)^{\frac{1}{\alpha}} z^{-\frac{1-\alpha}{\alpha}}\right)
$$

Hence, to generate $(Z, U)$, we could choose a bivariate envelope $(\tilde{Z}, \tilde{U})$ on $[0, \infty) \times[0, \pi]$ with density

$$
\tilde{p}(z, u)=\frac{z^{(1-\alpha) \lambda^{\alpha}-\frac{(1-\alpha) \nu}{\alpha}} e^{-z}}{\pi \Gamma\left((1-\alpha) \lambda^{\alpha}-\frac{(1-\alpha) \nu}{\alpha}+1\right)}
$$

and we have

$$
\begin{aligned}
\frac{f(z, u)}{\tilde{p}(z, u)} & =\frac{\Gamma\left((1-\alpha) \lambda^{\alpha}-\frac{(1-\alpha) \nu}{\alpha}+1\right) e^{\lambda^{\alpha}}}{G(\nu, \lambda)} B(u)^{\frac{\nu}{\alpha}} z^{-(1-\alpha) \lambda^{\alpha}} \exp \left(-\lambda B(u)^{\frac{1}{\alpha}} z^{-\frac{1-\alpha}{\alpha}}\right) \\
& \leq \frac{\Gamma\left((1-\alpha) \lambda^{\alpha}-\frac{(1-\alpha) \nu}{\alpha}+1\right) e^{(1-\alpha) \lambda^{\alpha}}}{G(\nu, \lambda)} \lambda^{-\alpha(1-\alpha) \lambda^{\alpha}} \alpha^{\alpha \lambda^{\alpha}}\left[(1-\alpha)^{(1-\alpha)} \alpha^{\alpha}\right]^{-\frac{\alpha \lambda^{\alpha}-\nu}{\alpha}} \\
& \leq \frac{C_{2}(\alpha, \lambda) \Gamma\left((1-\alpha) \lambda^{\alpha}-\frac{(1-\alpha) \nu}{\alpha}+1\right) \alpha^{\nu}(1-\alpha)^{\frac{(1-\alpha) \nu}{\alpha}}}{G(\nu, \lambda) \Gamma\left((1-\alpha) \lambda^{\alpha}+1\right)}=\bar{C}_{2}
\end{aligned}
$$

where $C_{2}(\alpha, \lambda)$ is defined in (3.13).

Alternatively, we could choose a bivariate envelope $(\hat{Z}, \hat{U})$ on $[0, \infty) \times[0, \pi]$ with density

$$
\hat{q}(z, u)=\frac{z^{(1-\alpha) \lambda^{\alpha}-\frac{(1-\alpha) \nu}{\alpha}} e^{-z}}{\Gamma\left((1-\alpha) \lambda^{\alpha}-\frac{(1-\alpha) \nu}{\alpha}+1\right)} \frac{\sqrt{2}}{\operatorname{Erf}\left(\pi / \sqrt{2 \sigma^{2}}\right) \sqrt{\pi \sigma^{2}}} e^{-\frac{u^{2}}{2 \sigma^{2}}}
$$

with $\sigma^{2}=\left[(1-\alpha)\left(\alpha \lambda^{\alpha}-\nu\right)\right]^{-1}$, i.e., we have

$\hat{Z} \sim \Gamma\left((1-\alpha) \lambda^{\alpha}-\frac{(1-\alpha) \nu}{\alpha}+1,1\right), \quad \hat{U} \sim \mathcal{N}\left(\mu=0, \sigma^{2}=\frac{1}{(1-\alpha)\left(\alpha \lambda^{\alpha}-\nu\right)}, \mathrm{lb}=0, \mathrm{ub}=\pi\right)$

Hence, according to the inequality (3.14), we have

$$
\begin{aligned}
\frac{f(z, u)}{\hat{q}(z, u)}= & \frac{\operatorname{Erf}\left(\pi \sqrt{(1-\alpha)\left(\alpha \lambda^{\alpha}-\nu\right) / 2}\right) \Gamma\left((1-\alpha) \lambda^{\alpha}-\frac{(1-\alpha) \nu}{\alpha}+1\right)}{G(\nu, \lambda) \sqrt{2 \pi(1-\alpha)\left(\alpha \lambda^{\alpha}-\nu\right)}} \\
& \times e^{\lambda^{\alpha}} B(u)^{\frac{\nu}{\alpha}} z^{-(1-\alpha) \lambda^{\alpha}} \exp \left(-\lambda B(u)^{\frac{1}{\alpha}} z^{-\frac{1-\alpha}{\alpha}}+\frac{(1-\alpha)\left(\alpha \lambda^{\alpha}-\nu\right) u^{2}}{2}\right) \\
\leq & \frac{\operatorname{Erf}\left(\pi \sqrt{(1-\alpha)\left(\alpha \lambda^{\alpha}-\nu\right) / 2}\right) \Gamma\left((1-\alpha) \lambda^{\alpha}-\frac{(1-\alpha) \nu}{\alpha}+1\right)}{G(\nu, \lambda) \Gamma\left((1-\alpha) \lambda^{\alpha}+1\right) \sqrt{2 \pi(1-\alpha)\left(\alpha \lambda^{\alpha}-\nu\right)}} \\
& \times(1-\alpha)^{\frac{(1-\alpha) \nu}{\alpha}} \alpha^{\nu} \Gamma\left((1-\alpha) \lambda^{\alpha}+1\right)(1-\alpha)^{-(1-\alpha) \lambda^{\alpha}} \lambda^{-\alpha(1-\alpha) \lambda^{\alpha}} e^{(1-\alpha) \lambda^{\alpha}}
\end{aligned}
$$




$$
\leq \frac{C_{4}(\alpha, \lambda) \Gamma\left((1-\alpha) \lambda^{\alpha}-\frac{(1-\alpha) \nu}{\alpha}+1\right)(1-\alpha)^{\frac{(1-\alpha) \nu}{\alpha}} \alpha^{\nu}}{G(\nu, \lambda) \Gamma\left((1-\alpha) \lambda^{\alpha}+1\right) \sqrt{1-\frac{\nu}{\alpha \lambda^{\alpha}}}}=\bar{C}_{4},
$$

where $\bar{C}_{4}$ is the associated $\mathrm{A} / \mathrm{R}$ constant. In general, given parameters $\alpha, \lambda, \nu$, we choose the envelope with the smallest $\mathrm{A} / \mathrm{R}$ constant to generate $G_{\alpha, \lambda, \nu}$. A combination of these four simulation schemes leads to a more efficient algorithm.

In general, the additional parameter $\nu$ for the GTS distributions makes analysing the complexity of Algorithm 4.1 more challenging, as the analytical form for $G(\nu, \lambda)$ is unknown. In the literature, the only existing algorithm for GTS distributions is the decomposition scheme (DS) proposed by Favaro et al. (2015). The relevant numerical comparison tests between Algorithm 4.1 and the DS scheme (Favaro et al., 2015) will be illustrated in Section 5.

\section{Numerical Verification and Comparison}

In this section, we provide numerical examples for sampling two families of tilted stable distributions: ETS and GTS distributions. The simulation experiments are all conducted on a normal laptop with the Intel Core i7-6500U CPU@2.50GHz processor, 8.00GB RAM, Windows 10 Home and 64-bit Operating System. The algorithms are coded and performed in R.3.4.2, and computing time is measured by the elapsed CPU time in seconds. Numerical validation and tests for the ETS algorithm are based on the probability density function (PDF), cumulative distribution function (CDF), and quantiles of $S_{\alpha, \lambda}$, which can be obtained by inverting Laplace transform (2.3) numerically. For the GTS simulation scheme, verifying via the CDF, PDF, and quantiles are nonexecutable as its closed-form Laplace transform is not available. So we establish comparison tests for the empirical CDFs, PDFs, and quantiles generated by Algorithm 4.1 and by the decomposition scheme (DS) of Favaro et al. (2015).

For Algorithm 3.1 of ETS distributions, the plots of CDFs and PDFs under parameter settings $\alpha=0.3,0.6, \lambda=1.0,5.0$ are provided in Figure 3. The Q-Q plots for the empirical quantiles of $S_{\alpha, \lambda}$ against the corresponding theoretical quantiles are presented in Figure 4, and the associated results in detail are reported in Table 1 . We can see that our algorithm can achieve a very high level of accuracy, and the simulated CDFs, PDFs, and quantiles are fitted pretty well to the associated numerical inversions. There are a variety of available algorithms for numerically inverting Laplace transforms with high accuracy in the literature, such as Gaver (1966), Stehfest (1970) and Abate and Whitt $(1992,1995,2006)$ to name a few. Here, we adopt the classical Euler scheme as described in Abate and Whitt (2006, Section 5, p.415-416). 
Table 1: Comparison of the empirical quantiles of $S_{\alpha, \lambda}$ for the SR scheme (via Algorithm 3.1) against the theoretical quantiles of $S_{\alpha, \lambda}$ approximated via numerical inverse the Laplace transform of (2.3)

\begin{tabular}{c|ccccccccr}
\hline \hline Quantile & $10 \%$ & $20 \%$ & $30 \%$ & $40 \%$ & $50 \%$ & $60 \%$ & $70 \%$ & $80 \%$ & $90 \%$ \\
\hline \hline & & & & $\alpha=0.3$ & $\lambda=1$ & & & & \\
\hline 2D SR & 0.0172 & 0.0342 & 0.0566 & 0.0877 & 0.1299 & 0.1912 & 0.2874 & 0.4451 & 0.7754 \\
Numerical Inverse & 0.0173 & 0.0342 & 0.0567 & 0.0877 & 0.1303 & 0.1913 & 0.2873 & 0.4452 & 0.7756 \\
\hline \hline & & & & $\alpha=0.6$ & $\lambda=5$ & & & & \\
\hline 2D SR & 0.1592 & 0.1905 & 0.2185 & 0.2466 & 0.2773 & 0.3125 & 0.3558 & 0.4163 & 0.5181 \\
Numerical Inverse & 0.1592 & 0.1905 & 0.2184 & 0.2466 & 0.2772 & 0.3125 & 0.3562 & 0.4163 & 0.5182 \\
\hline \hline
\end{tabular}
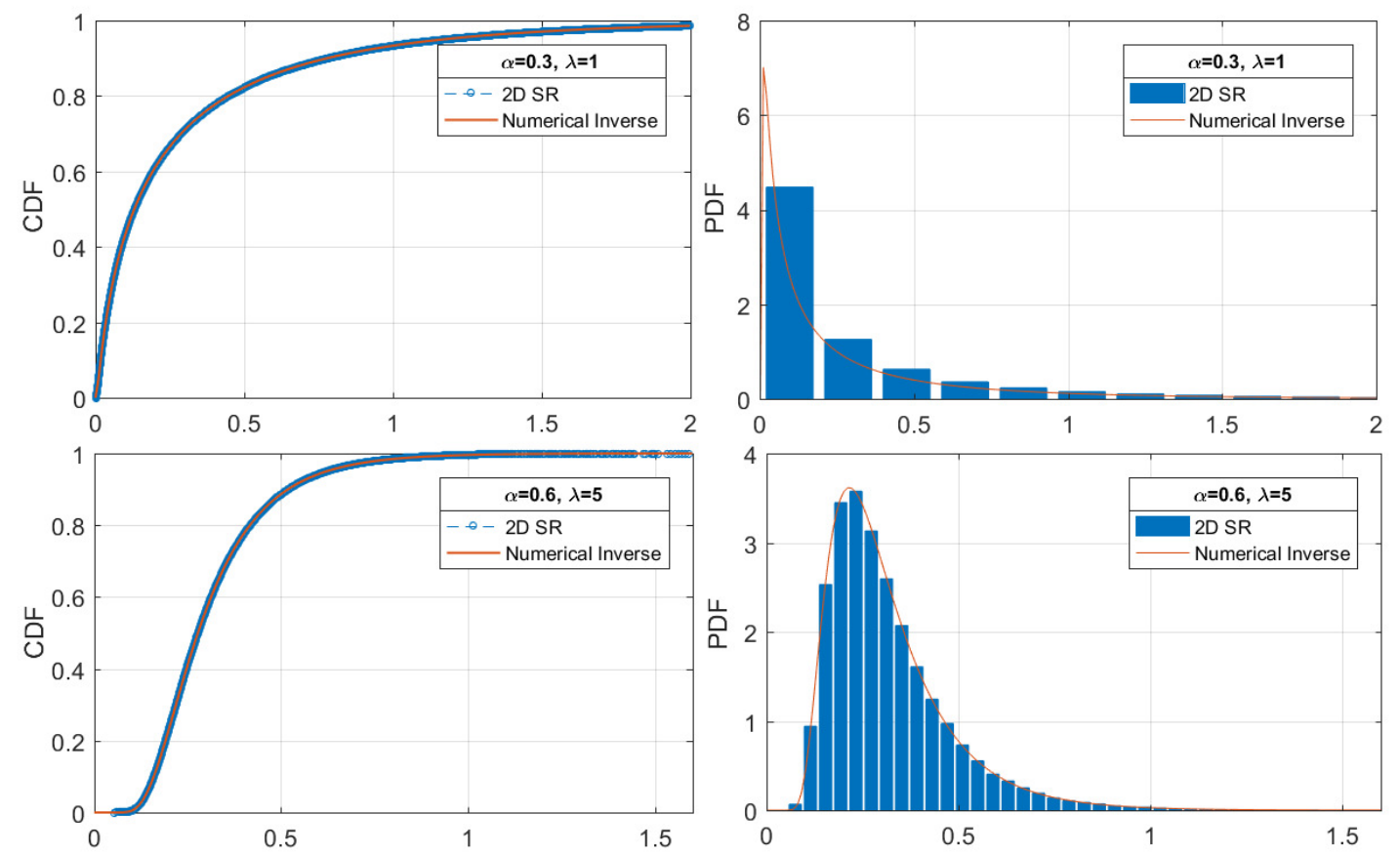

Figure 3: Comparison of the empirical CDF/PDF for the SR scheme (via Algorithm 3.1) of $S_{\alpha, \lambda}$ with the $\mathrm{CDF} / \mathrm{PDF}$ obtained via numerical inverse the Laplace transform of (2.3)
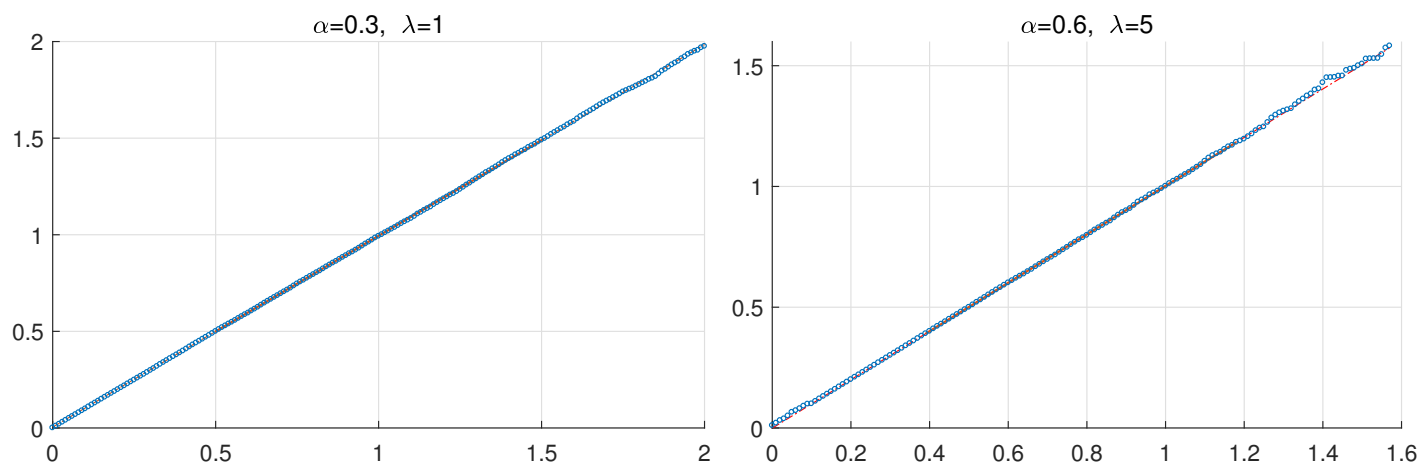

Figure 4: Q-Q plots with the vertical axis being the empirical quantiles of $S_{\alpha, \lambda}$ for the SR scheme (via Algorithm 3.1) and the horizonal axis being the theoretical quantiles of $S_{\alpha, \lambda}$ approximated via numerical inverse the Laplace transform of (2.3) 
To investigate the performance of our SR scheme for the ETS, we made a comparison of the CPU time for Algorithm 3.1 against the DR scheme for simulating 100, 000 samples under the parameter settings $\alpha \in\{0.05,0.1, \ldots, 0.9,0.99\}$ and $\lambda \in\left\{0.01,0.1, \ldots, 10^{6}\right\}$. The numerical results are reported in Table 2. We can see that our SR scheme performances well for all combinations of $\alpha$ and $\lambda$. The out-performance of our algorithm would even become much more substantial when $\alpha$ is close to 0 . For example, it is about 8 times faster than the DR scheme when $\alpha=0.05$. In addition, Algorithm 3.1 is also very fast when the tilting parameter $\lambda$ is not very large, which clearly indicates that the acceptance rate of Algorithm 3.1 is higher than the DR scheme (Devroye, 2009) for a small tilting parameter $\lambda$. Based on the DR scheme, Hofert (2011b) proposed a more efficient sampling algorithm for ETS distributions by combining the FR scheme with the DR scheme. Since the SR scheme outperforms the DR scheme over all combination of parameters, this combination algorithms in Hofert (2011b) can be further improved by combining the SR scheme with the FR scheme.

Table 2: Comparison of CPU time for generating 100, 000 samples based on the SR scheme (via Algorithm 3.1) and the DR scheme (Devroye, 2009), respectively

\begin{tabular}{|c|c|c|c|c|c|c|c|c|c|c|c|c|c|c|c|c|c|c|}
\hline & \multicolumn{2}{|c|}{0.01} & \multicolumn{2}{|c|}{0.10} & \multicolumn{2}{|c|}{1.00} & \multicolumn{2}{|c|}{10} & \multicolumn{2}{|c|}{100} & \multicolumn{2}{|c|}{1,000} & \multicolumn{2}{|c|}{10,000} & \multicolumn{2}{|c|}{100,000} & \multicolumn{2}{|c|}{$1,000,000$} \\
\hline & 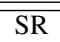 & $\overline{\overline{D R}}$ & 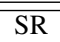 & 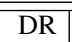 & 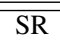 & $\overline{\mathrm{DR}}$ & 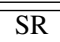 & $\overline{\mathrm{DR}}$ & 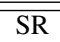 & 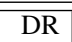 & 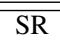 & $\overline{\overline{\mathrm{DR}}}$ & 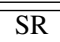 & 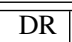 & 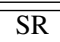 & 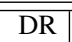 & $\overline{\overline{\text { SR }}}$ & $\overline{\bar{D}}$ \\
\hline 0.05 & 2.58 & 235 & 2.33 & 8.80 & 2.36 & 1005 & 2.42 & 19.24 & 2.23 & 18.62 & 2.22 & 19.09 & 2.47 & 18.79 & 2.32 & 18.59 & 45 & 18.16 \\
\hline 0.1 & 2.51 & 6 & 67 & 1 & 2.56 & 1 & 2.47 & & 2.62 & & 4.44 & & 3.96 & 17 & 4.08 & 17.3 & 4 & 16. \\
\hline & 2. & & 53 & & 22 & & 51 & & 6 & & 3.50 & & 58 & & 22 & & 5 & 5.18 \\
\hline & & & 2.36 & & 45 & & & & 3.9 & & & & & & & & & 4.0 \\
\hline 0. & 1. & & 2.35 & 1 & 4.03 & 3 & 3.86 & & 3.69 & 7. & 3.78 & & 3.47 & 4.6 & 3.19 & 4. & U & 4.2 \\
\hline 0. & 1.73 & 1.5 & 94 & 8.53 & .59 & 1 & 3.50 & 3 & 3.22 & 5.1 & 3.11 & 4. & 3.36 & 4.2 & .53 & 3. & 69 & 4.0 \\
\hline 0.6 & 1.56 & 6 & 1.97 & 1 & 3.65 & 1 & 3.28 & 7 & 3.39 & 4. & 3.19 & & 3.00 & 4. & 49 & 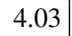 & 7 & 4.0 \\
\hline & 1.61 & & 1.76 & & 3.46 & & 3.17 & & 3.01 & & 3. & & $J$. & & & & & 4.0 \\
\hline & & & 1. & & 3. & & 2. & & 2. & & & & & & & & & \\
\hline & & & 1.9 & & 1.7 & & 2.90 & & 2.76 & & 2. & & 2.8 & & & & & 4.97 \\
\hline 0. & 1.50 & 17.81 & 1.54 & 18.00 & 1.62 & 6 & 1.88 & 18.44 & 3.14 & 13.94 & 2.28 & 4.41 & 2.64 & 4.69 & 3.02 & 4.06 & 2.83 & 4.21 \\
\hline
\end{tabular}

The comparison of empirical CDFs and CDFs for Algorithm 4.1 and the DS scheme under various combinations of $(\alpha, \lambda, \nu)$ are illustrated in Figure 5. We also present the comparison of empirical quantiles in Figure 6, and report the associated results in Table 3. We can see that these two algorithms are closely matched in terms of CDF, PDF and quantiles. Note that, Algorithm 4.1 also has one special feature, that is, it can be used to sample $G_{\alpha, \lambda, \nu}$ for a negative $\nu$ such that $\nu>-\alpha \lambda^{\alpha}$. Figure 7 demonstrates the distributional behaviour of this special class of GTS via its PDFs.

Meanwhile, we have also compared the simulation time for Algorithm 4.1 against the decomposition scheme over a large range of values of $\alpha, \lambda, \nu$, and explore how the efficiency depends on them. The related numerical results are listed in Table 4 . We see that our scheme is more efficient 

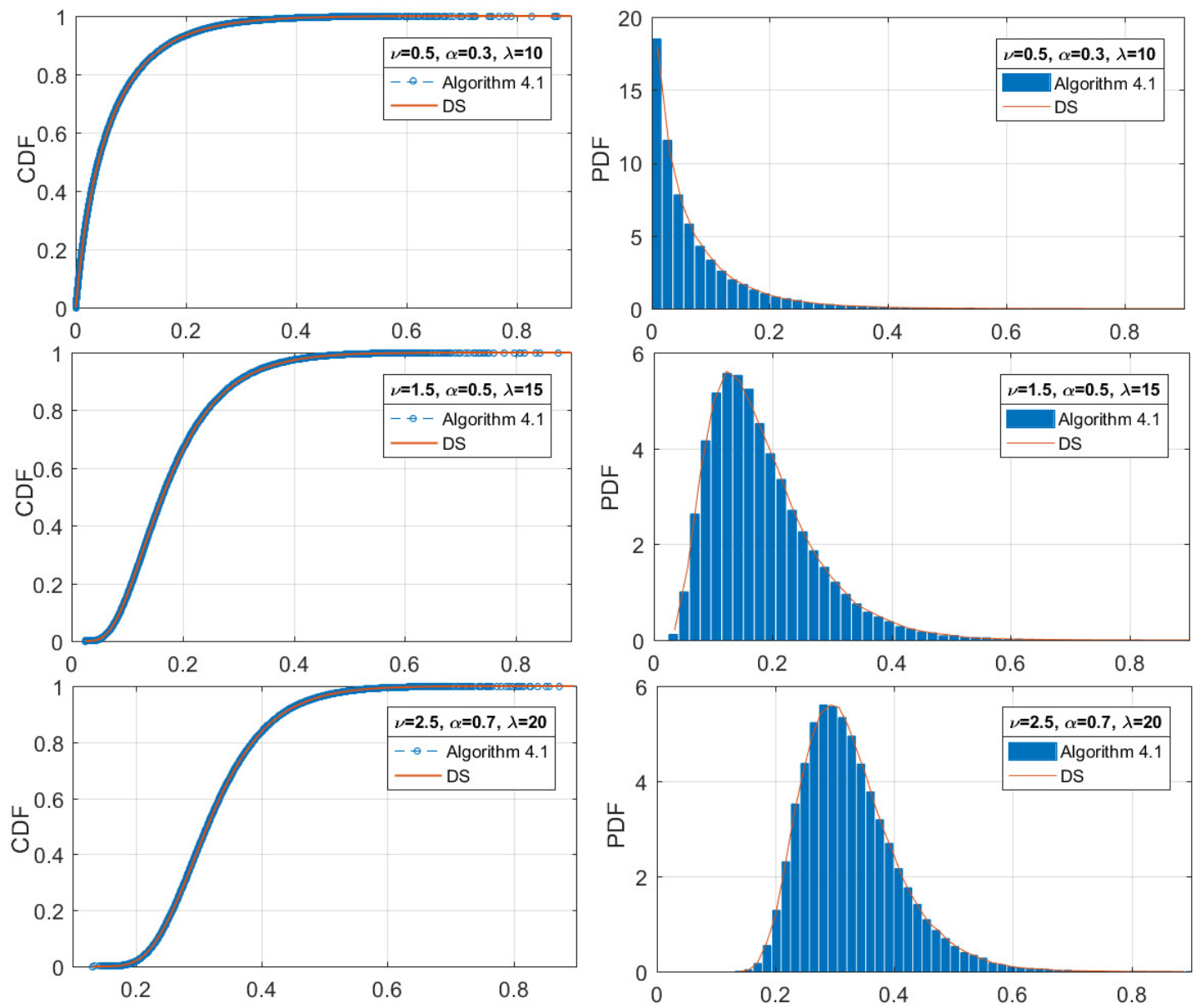

Figure 5: Comparison of the empirical CDF/PDF for Algorithm 4.1 against the decomposition scheme (DS) (Favaro et al., 2015)
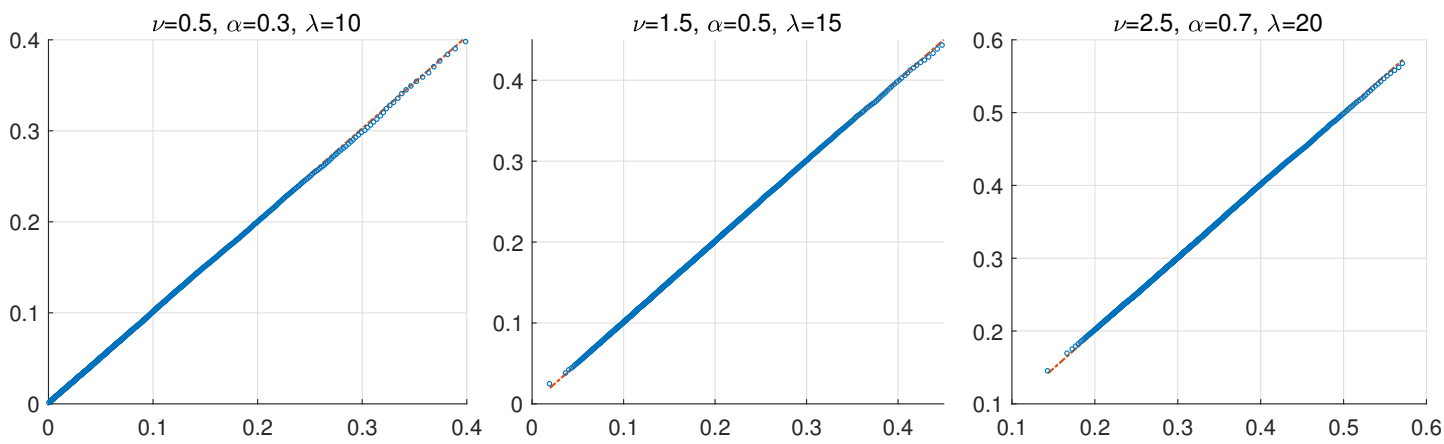

Figure 6: Q-Q plots with the vertical axis being the empirical quantiles of $G_{\alpha, \lambda, \nu}$ for Algorithm 4.1 and the horizonal axis being the empirical quantiles of $G_{\alpha, \lambda, \nu}$ for the decomposition scheme (DS) (Favaro et al., 2015)

for most parameter settings provided in Table 4, especially for large values of $\alpha, \lambda$. For example, Algorithm 4.1 is extraordinarily fast when $\alpha=0.6$ and $\lambda=1,000$. In general, our proposed algorithm is significantly more efficient for a large range of parameter combinations. The key reason is that our SR scheme is developed independently and generates the GTS random variable directly 
Table 3: Comparison of the empirical quantiles of $G_{\alpha, \lambda, \nu}$ for Algorithm 4.1 against the decomposition scheme (DS) (Favaro et al., 2015)

\begin{tabular}{c|ccccccccc}
\hline \hline Quantile & $10 \%$ & $20 \%$ & $30 \%$ & $40 \%$ & $50 \%$ & $60 \%$ & $70 \%$ & $80 \%$ & $90 \%$ \\
\hline \hline & & & & $\nu=0.5$ & $\alpha=0.3$ & $\lambda=10$ & & & \\
\hline Algo 4.1 & 0.0164 & 0.0271 & 0.0384 & 0.0510 & 0.0660 & 0.0840 & 0.1079 & 0.1420 & 0.2013 \\
DS & 0.0165 & 0.0272 & 0.0384 & 0.0509 & 0.0660 & 0.0844 & 0.1079 & 0.1419 & 0.2012 \\
\hline \hline & & & & $\nu=1.5$ & $\alpha=0.5$ & $\lambda=15$ & & & \\
\hline Algo 4.1 & 0.0871 & 0.1081 & 0.1263 & 0.1441 & 0.1632 & 0.1849 & 0.2107 & 0.2451 & 0.3001 \\
DS & 0.0871 & 0.1083 & 0.1263 & 0.1443 & 0.1631 & 0.1849 & 0.2107 & 0.2450 & 0.3001 \\
\hline \hline & & & & $\nu=2.5$ & $\alpha=0.7$ & $\lambda=20$ & & & \\
\hline Algo 4.1 & 0.2353 & 0.2583 & 0.2776 & 0.2955 & 0.3138 & 0.3336 & 0.3566 & 0.3861 & 0.4315 \\
DS & 0.2352 & 0.2583 & 0.2779 & 0.2954 & 0.3140 & 0.3334 & 0.3569 & 0.3862 & 0.4312 \\
\hline \hline
\end{tabular}
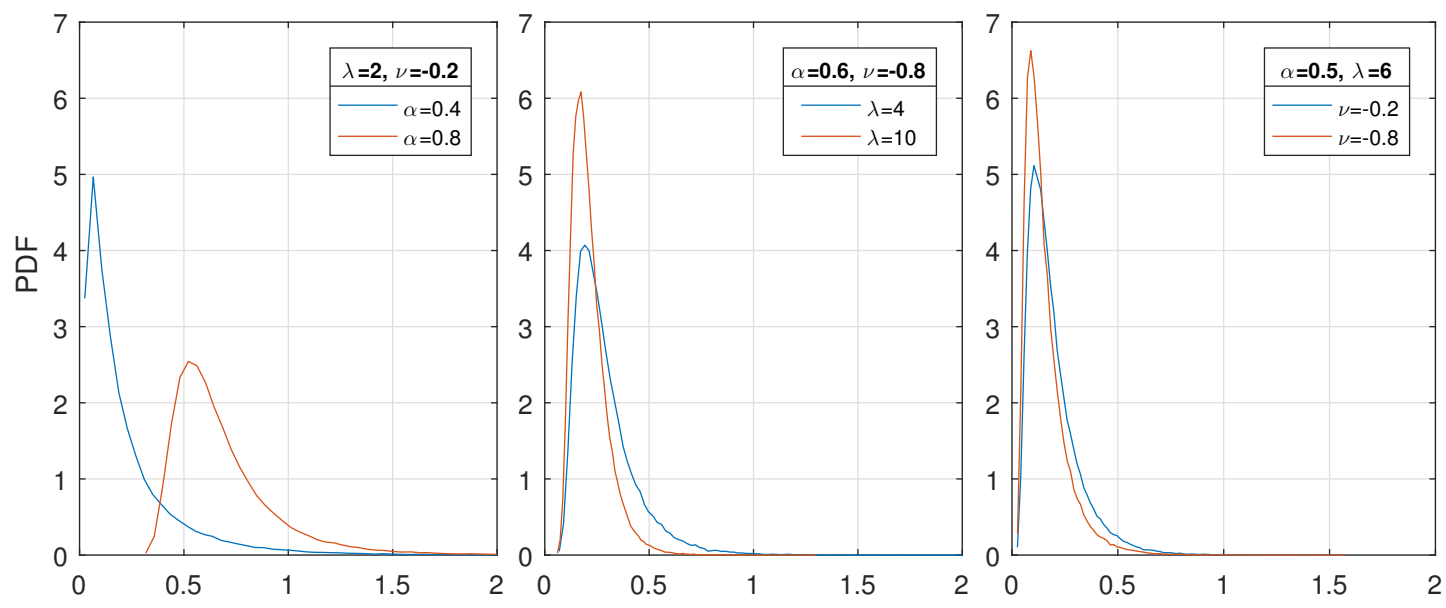

Figure 7: PDFs of GTS with $\nu<0$

without using the DR or FR method. This leads to a more straightforward procedure for implementation. In fact, the DS scheme can be improved by generating the ETS random variable using our Algorithm 3.1, which would then speed up the entire simulation for the GTS random variable.

\section{Conclusion}

In this paper, a new efficient simulation scheme has been developed for sampling exponential tilted stable and gamma tilted stable distributions. The two important distributions appear routinely in financial applications and other areas that heavily rely on Monte Carlo simulation. The key principle of this approach is two-dimensional single rejection, which is very different from other existing schemes in the literature. The complexity of our new algorithm for the ETS family is uniformly bounded over all ranges of parameters. Remarkably, it beats all other algorithms. Our further extension for exactly sampling the GTS family does not rely on sampling the ETS family, hence, our algorithm for the GTS family is more efficient than the decomposition scheme (which is the only alternative algorithm in the literature). For future research, our algorithms can be adopted for further 
Table 4: Comparison of CPU time for generating 100, 000 samples based on Algorithm 4.1 and the decomposition scheme (DS) (Favaro et al., 2015), respectively

\begin{tabular}{|c|c|c|c|c|c|c|c|c|c|c|c|c|c|c|}
\hline \multirow[b]{2}{*}{$\lambda$} & \multicolumn{2}{|c|}{$\nu=1$} & \multicolumn{2}{|c|}{$\nu=1.5$} & \multicolumn{2}{|c|}{$\nu=2$} & \multicolumn{2}{|c|}{$\nu=2.5$} & \multicolumn{2}{|c|}{$\nu=3$} & \multicolumn{2}{|c|}{$\nu=3.5$} & \multicolumn{2}{|c|}{$\nu=4$} \\
\hline & Algo 4.1 & DS & Algo 4.1 & DS & Algo 4.1 & DS & Algo 4.1 & DS & Algo 4.1 & DS & Algo 4.1 & DS & Algo 4.1 & $\overline{\mathrm{DS}}$ \\
\hline \multicolumn{15}{|c|}{$\alpha=0.2$} \\
\hline 10 & & 89 & 56 & 8.31 & 81 & 12.57 & 5.45 & 12.44 & 7 & 16.17 & 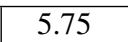 & 16.71 & 5.95 & 0.77 \\
\hline 100 & 58 & 48 & 3.76 & 7.81 & 5 & 12.19 & 4.56 & 12.84 & 4.54 & 17.58 & 4.82 & 16.80 & 4.75 & 21.0 \\
\hline 000 & 3.25 & 7.22 & 3.35 & 8.07 & 3.43 & 12.33 & 3.45 & 13.05 & 5.92 & 16.76 & 4.25 & 18.12 & 4.35 & 21.7 \\
\hline \multicolumn{15}{|c|}{$\overline{\alpha=0.4}$} \\
\hline 10 & 43 & 7.02 & 4.67 & 7.98 & .56 & 12.97 & 6.43 & 13.95 & 6.89 & 17.14 & 7.80 & 17.70 & 8.64 & 21.11 \\
\hline 100 & 5 & 6.88 & 6 & 7.79 & 5 & 12.89 & 4. & 13.08 & 7 & 16.97 & 5.23 & 16.64 & 5.54 & 21.0 \\
\hline 1,000 & 3.28 & 6.81 & 3.49 & 8.88 & 3.34 & 11.89 & 3.54 & 13.88 & 3.53 & 16.31 & 3.89 & 18.14 & 4.23 & 21.0 \\
\hline \multicolumn{15}{|c|}{$\alpha=0.6$} \\
\hline 10 & 53 & 7.27 & 5.42 & 7.89 & 6.32 & 12.89 & 7.35 & 13.00 & .23 & 16.89 & 10.08 & 17.16 & $11.82 \quad 2$ & 21.44 \\
\hline 100 & 23 & 6.72 & 4.23 & 12.28 & 13 & 12.08 & 4.23 & 14.22 & 4.13 & 16.44 & 4.56 & 17.64 & 5.23 & 20.8 \\
\hline, 000 & 3.44 & 7.73 & 3.45 & 1540.32 & 3.25 & 12.16 & 3.62 & 186.15 & 3.15 & 16.52 & 3.86 & 54.73 & 3.65 & 20.67 \\
\hline
\end{tabular}

generating ETS-driven or GTS-driven stochastic processes as mentioned early in the introduction, which could lead many simulation-based applications.

\section{References}

Abate, J. and Whitt, W. (1992). The Fourier-series method for inverting transforms of probability distributions. Queueing Systems, 10(1):5-87.

Abate, J. and Whitt, W. (1995). Numerical inversion of Laplace transforms of probability distributions. ORSA Journal on Computing, 7(1):36-43.

Abate, J. and Whitt, W. (2006). A unified framework for numerically inverting Laplace transforms. INFORMS Journal on Computing, 18(4):408-421.

Andrieu, C., Doucet, A., and Holenstein, R. (2010). Particle Markov chain Monte Carlo methods. Journal of the Royal Statistical Society: Series B (Statistical Methodology), 72(3):269-342.

Barndorff-Nielsen, O. E. and Shephard, N. (2001). Normal modified stable processes. Theory of Probability and Mathematical Statistics, pages 1-19.

Barndorff-Nielsen, O. E. and Shephard, N. (2002). Econometric analysis of realized volatility and its use in estimating stochastic volatility models. Journal of the Royal Statistical Society: Series B (Statistical Methodology), 64(2):253-280.

Barndorff-Nielsen, O. E. and Shephard, N. (2003). Integrated OU processes and non-Gaussian OU-based stochastic volatility models. Scandinavian Journal of Statistics, 30(2):277-295.

Batir, N. (2008). Inequalities for the gamma function. Archiv der Mathematik, 91(6):554-563.

Brix, A. (1999). Generalized gamma measures and shot-noise Cox processes. Advances in Applied Probability, 31(4):929-953. 
Carr, P. and Wu, L. (2003). The finite moment log stable process and option pricing. Journal of Finance, 58(2):753-777.

Dassios, A., Lim, J. W., and Qu, Y. (2020). Exact simulation of truncated Lévy subordinator. ACM Transactions on Modeling and Computer Simulation, 30(3):17:1-17:26.

Dassios, A., Qu, Y., and Zhao, H. (2018). Exact simulation for a class of tempered stable and related distributions. ACM Transactions on Modeling and Computer Simulation, 28(3):20:1-20:21.

Devroye, L. (2009). Random variate generation for exponentially and polynomially tilted stable distributions. ACM Transactions on Modeling and Computer Simulation, 19(4):1-20.

Favaro, S., Nipoti, B., and Teh, Y. W. (2015). Random variate generation for Laguerre-type exponentially tilted $\alpha$-stable distributions. Electronic Journal of Statistics, 9(1):1230-1242.

Gaver, J. D. P. (1966). Observing stochastic processes, and approximate transform inversion. Operations Research, 14(3):444-459.

Hofert, M. (2011a). Efficiently sampling nested Archimedean copulas. Computational Statistics \& Data Analysis, 55(1):57-70.

Hofert, M. (2011b). Sampling exponentially tilted stable distributions. ACM Transactions on Modeling and Computer Simulation, 22(1):1-11.

Hougaard, P. (1986). Survival models for heterogeneous populations derived from stable distributions. Biometrika, 73(2):387-396.

Law, A. M. (2015). Simulation Modeling and Analysis. McGraw-Hill Education, New York, 5 edition.

Lévy, P. (1925). Calcul des Probabilités. Gauthier-Villars, Paris.

Li, L. and Linetsky, V. (2013). Optimal stopping and early exercise: an eigenfunction expansion approach. Operations Research, 61(3):625-643.

Li, L. and Linetsky, V. (2014). Time-changed Ornstein-Uhlenbeck processes and their applications in commodity derivative models. Mathematical Finance, 24(2):289-330.

Li, L. and Linetsky, V. (2015). Discretely monitored first passage problems and barrier options: an eigenfunction expansion approach. Finance and Stochastics, 19(4):941-977.

Mandelbrot, B. (1961). Stable Paretian random functions and the multiplicative variation of income. Econometrica, 29(4):517-543.

Mandelbrot, B. (1963a). New methods in statistical economics. Journal of Political Economy, 71(5):421440.

Mandelbrot, B. (1963b). The variation of certain speculative prices. Journal of Business, 36(4):394-419. 
Mendoza-Arriaga, R. and Linetsky, V. (2014). Time-changed CIR default intensities with two-sided meanreverting jumps. Annals of Applied Probability, 24(2):811-856.

Mendoza-Arriaga, R. and Linetsky, V. (2016). Multivariate subordination of Markov processes with financial applications. Mathematical Finance, 26(4):699-747.

Nishiyama, Y. and Fukumizu, K. (2016). Characteristic kernels and infinitely divisible distributions. The Journal of Machine Learning Research, 17(1):6240-6267.

Qu, Y., Dassios, A., and Zhao, H. (2019). Efficient simulation of Lévy-driven point processes. Advances in Applied Probability, 51(4):927-966.

Qu, Y., Dassios, A., and Zhao, H. (2021). Exact simulation of Ornstein-Uhlenbeck tempered stable processes. Journal of Applied Probability, 58(2).

Stehfest, H. (1970). Algorithm 368: Numerical inversion of Laplace transforms. Communications of the ACM, 13(1):47-49.

Todorov, V. (2015). Jump activity estimation for pure-jump semimartingales via self-normalized statistics. Annals of Statistics, 43(4):1831-1864.

Tweedie, M. (1984). An index which distinguishes between some important exponential families. In Statistics: Applications and New Directions: Proceedings of the Indian Statistical Institute Golden Jubilee International Conference, pages 579-604.

Wu, L. (2006). Dampened power law: reconciling the tail behavior of financial security returns. Journal of Business, 79(3):1445-1473.

Zolotarev, V. M. (1966). On representation of stable laws by integrals. In Selected Translations in Mathematical Statistics and Probability, volume 6, pages 84-88. American Mathematical Society.

Zolotarev, V. M. (1986). One-dimensional Stable Distributions. American Mathematical Society, Providence. 